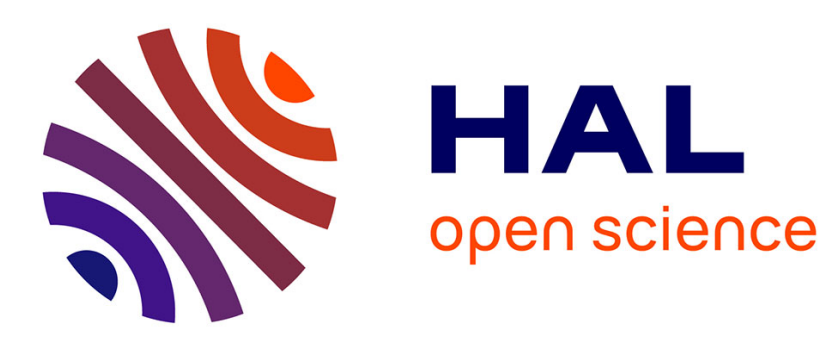

\title{
Phase Transitions of the $k$-Majority Dynamics in a Biased Communication Model
}

Emilio Cruciani, Hlafo Alfie Mimun, Matteo Quattropani, Sara Rizzo

\section{To cite this version:}

Emilio Cruciani, Hlafo Alfie Mimun, Matteo Quattropani, Sara Rizzo. Phase Transitions of the $k$-Majority Dynamics in a Biased Communication Model. ICDCN 2021 - 22nd International Conference on Distributed Computing and Networking, Jan 2021, Nara / Virtual, Japan. pp.146-155, 10.1145/3427796.3427811. hal-03007242

\section{HAL Id: hal-03007242 \\ https://hal.science/hal-03007242}

Submitted on 20 Jan 2021

HAL is a multi-disciplinary open access archive for the deposit and dissemination of scientific research documents, whether they are published or not. The documents may come from teaching and research institutions in France or abroad, or from public or private research centers.
L'archive ouverte pluridisciplinaire HAL, est destinée au dépôt et à la diffusion de documents scientifiques de niveau recherche, publiés ou non, émanant des établissements d'enseignement et de recherche français ou étrangers, des laboratoires publics ou privés. 


\title{
Phase Transitions of the $k$-Majority Dynamics in a Biased Communication Model
}

\author{
Emilio Cruciani \\ Inria, I3S Lab, UCA, CNRS \\ Sophia Antipolis, France \\ emilio.cruciani@inria.fr \\ Matteo Quattropani \\ LUISS Guido Carli \\ Rome, Italy \\ mquattropani@luiss.it
}

\author{
Hlafo Alfie Mimun \\ LUISS Guido Carli \\ Rome, Italy \\ hmimun@luiss.it \\ Sara Rizzo \\ Gran Sasso Science Institute \\ L'Aquila, Italy \\ sara.rizzo@gssi.it
}

\begin{abstract}
Consider a graph where each of the $n$ nodes is in one of two possible states. Herein, we analyze the synchronous $k$-MAJORITY dynamics, where nodes sample $k$ neighbors uniformly at random with replacement and adopt the majority state among the nodes in the sample (potential ties are broken uniformly at random). This class of dynamics generalizes other well-known dynamics, e.g., VOTER and 3-MAJORITY, which have been studied in the literature as distributed algorithms for consensus.

We consider a biased communication model: whenever nodes sample a neighbor they see state $\sigma$ with some probability $p$, regardless of the state of the sampled node, and its true state with probability $1-p$. Differently from previous works where specific graph topologies - typically characterized by good expansion properties - are considered, our analysis only requires the graphs to be sufficiently dense, i.e., to have minimum degree $\omega(\log n)$, without any further topological assumption.

In this setting we prove two phase transition phenomena, both occurring with high probability, depending on the bias $p$ and on the initial unbalance toward state $\sigma$. More in detail, we prove that for every $k \geq 3$ there exists a $p_{k}^{\star}$ such that if $p>p_{k}^{\star}$ the process reaches in $\mathcal{O}(1)$ rounds a $\sigma$-almost-consensus, i.e., a configuration where a fraction $1-\gamma$ of the volume is in state $\sigma$, for any arbitrarily-small positive constant $\gamma$. On the other hand, if $p<p_{k}^{\star}$, we look at random initial configurations in which every node is in state $\sigma$ with probability $1-q$ independently of the others. We prove that there exists a constant $q_{p, k}^{\star}$ such that if $q<q_{p, k}^{\star}$ then a $\sigma$-almost-consensus is still reached in $\mathcal{O}(1)$ rounds, while, if $q>q_{p, k}^{\star}$, the process spends $n^{\omega(1)}$ rounds in a metastable phase where the fraction of volume in state $\sigma$ is around a constant value depending only on $p$ and $k$.

Finally we also investigate, in such a biased setting, the differences and similarities between $k$-MAJORITY and other closely-related dynamics, namely VOTER and DETERMINISTIC MAJORITY.
\end{abstract}




\section{Introduction}

Designing distributed algorithms that let the nodes of a graph reach a consensus, i.e., a configuration of states where all the nodes agree on the same state, is a fundamental problem in distributed computing and multi-agent systems. Consensus algorithms are used in protocols for other tasks, such as leader election and atomic broadcast, and in real-world applications such as clock synchronization tasks and blockchains.

Recently there has been a growing interest in the analysis of simple local dynamics as distributed algorithms for the consensus problem $\mathrm{BCN}^{+} 17, \mathrm{CGG}^{+}$18, $\mathrm{CER}^{2}, \mathrm{DGM}^{+} 11, \mathrm{GL18}$, HP01, MNT14, inspired by simple mechanisms studied in statistical mechanics for interacting particle systems Lig12. In this scenario, nodes are anonymous (i.e., they do not have distinct IDs) and they have a state that evolves over time according to some common local interaction with their neighbors.

The first dynamics investigated with this goal has been voTER [HP01, where nodes copy the state of a random neighbor. The authors prove that the dynamics reaches a consensus on state $\sigma$ with probability proportional to the volume of nodes initially in state $\sigma$ in $\mathcal{O}\left(n^{3} \log n\right)$ rounds, regardless of the graph structure. Recently the upper bound has been improved to $\mathcal{O}\left(n^{3}\right)$ [KMS19], which is shown to be tight. However, the dynamics is slow in reaching consensus, e.g., it needs $\Omega(n)$ rounds in the complete graph despite the extremely good connectivity properties of the topology. Therefore, simple generalizations of VOTER have been considered in order to achieve a faster distributed algorithm for consensus. One of the directions has been that of considering more than a single neighbor in the sample. For example, in the 3-MAJORITY dynamics, where each node samples 3 neighbors with replacement and updates its state to the most frequent state among those in the sample, the time needed to reach a consensus on the complete graph lowers from $\Omega(n)$ to $\mathcal{O}(\log n)$ CEOR13, GL18]. A more detailed discussion on related works about dynamics for consensus is deferred to Section 2.

\subsection{Our Contribution}

Herein we focus on the scenario in which every node has a binary state (either $\mathcal{R}$ or $\mathcal{B}$ ) and the communication among the nodes proceeds in synchronous rounds; in this setting we analyze the $k$-MAJORITY dynamics, where nodes update their state to that of the majority of a random sample of size $k$ of their neighborhood.

Obviously, starting from a monochromatic configuration, w.l.o.g., the one in which every node is in state $\mathcal{R}$, every vertex remains in the same initial state forever. Suppose now there are chances that some vertices change state because of some communication noise. A natural arising question is how strong has to be the noise to subvert the initial consensus?

In this paper we aim at providing a rigorous answer to such a non-rigorous question. We do so by considering - differently from most of the previous works - a communication model which is biased. W.l.o.g., the bias is toward state $\mathcal{B}$, i.e., whenever nodes sample a neighbor they see state $\mathcal{B}$ with some probability $p$, regardless of the state of the sampled node, and its true state with probability $1-p$.

This biased communication model has been introduced in [CNNS18, where the authors analyze the 2-CHOICES dynamics on core-periphery networks. We formally present the communication model in Section 3 and we discuss potential applications of this framework in Section 7. Other existing models of bias are discussed in Section 2 .

We stress that most of the previous works rely on strong topological assumptions, e.g., considering complete graphs or expanders, to prove upper bounds on the consensus time of the dynamics. We move a step forward in this direction by removing all assumptions that depend on the topology of the graph; indeed we only require the graph to be sufficiently dense, i.e., with 
minimum degree $\omega(\log n)$. Such a milder assumption, though, comes at the cost of a weaker notion of consensus, i.e., what we call almost-consensus (Definition 3.2). Informally, a process reaches an almost-consensus on a state $\sigma$ whenever a fraction $1-\gamma$ of the volume of the graph is in state $\sigma$, for any arbitrarily-small positive constant $\gamma$. Indeed, pushing our techniques to their limit, we could prove a consensus on state $\sigma$ in $\mathcal{O}(\log n)$ rounds. We would still require no topological assumptions, but just a stronger condition on the minimum degree (from $\omega(\log n)$ to $\Omega(n)$ ), thus dramatically restricting the class of graphs taken into account to extremely dense ones.

Note that, in the biased communication model we consider, the Markov Chain that models the process has a single absorbing state where all nodes are in state $\sigma$. For this reason, in Section 5.1 we first consider an initial configuration where none of the nodes is in state $\sigma$, and study the time needed by the process to reach a $\sigma$-almost-consensus. Trivially, if $p=0$ the process remains stuck in its initial configuration, while if $p=1$ the process reaches the absorbing state in one single round. More in general, it is intuitive that the process will converge slowly to the absorbing state if $p$ is small and quickly if $p$ is large. With such an intuition in mind, we prove a first phase transition phenomenon.

Informal description of Theorem 5.1. Consider the $k$-MAJORITY dynamics in the communication model with bias $p$ and where initially no nodes are in state $\sigma$.

For every $k \geq 3$ there exists a constant $p_{k}^{\star} \in\left[\frac{1}{9}, \frac{1}{2}\right]$ such that:

- If $p<p_{k}^{\star}$, then a $\sigma$-almost-consensus is reached in $n^{\omega(1)}$ rounds, w.h.p.

- If $p>p_{k}^{\star}$, then a $\sigma$-almost-consensus is reached in $\mathcal{O}(1)$ rounds, w.h.p.

In the proof, similarly to what has been done in CNNS18 for the 2-CHOICES dynamics, we look at the expected evolution of the fraction of neighbors in state $\sigma$ for every node of the graph. However, comparing the analysis with that of [CNNS18, here we have a more comprehensive scenario ( $k$-MAJORITY for any $k$ vs. 2-CHOICES) with substantially more precise results.

Another important innovation with respect to CNNS18 is the presence of another phase transition in the initial state that we discuss in Section 5.2. Clearly in the fast convergence regime, i.e., when $p>p_{k}^{\star}$, the initial configuration in which none of the nodes is in state $\sigma$ is the hardest one for the process to reach a $\sigma$-almost-consensus. However, the scenario changes in the slow convergence regime, where a different initial configuration with some of the nodes already in state $\sigma$ could be enough to change the behavior of the dynamics. In this more general scenario, we prove a second phase transition phenomenon.

Informal description of Theorem 5.7. Consider the $k$-MAJORITY dynamics in the communication model with bias $p<p_{k}^{\star}$ and where initially every node is in state $\sigma$ with probability $1-q$, independently of the others.

For every $k \geq 3$ there exists a constant $q_{p, k}^{\star}$ such that:

- If $q<q_{p, k}^{\star}$, then a $\sigma$-almost-consensus is reached in $\mathcal{O}(1)$ rounds, w.h.p.

- If $q>q_{p, k}^{\star}$, then a $\sigma$-almost-consensus is reached in $n^{\omega(1)}$ rounds, w.h.p.

Note that the notation $q_{p, k}^{\star}$ is different from the one appearing in Section 5.2 in order to make clearer this introductory exposition.

In Section 4 we prove essentially the same result on directed infinite trees. In that case the phase transition occurs on the probability of the root to be in the state not promoted by the 
bias as the number of rounds goes to infinity. The result in this "toy model" is presented before the others and used as a guideline along the paper: the intuitions behind the proof are the same as those of the previously presented results, but the calculations are much less technically involved.

Finally, in Section 6, we characterize the behavior of VOTER and of DETERMINISTIC MAJORITY, where nodes simply update to the majority state in their neighborhood, under the same biased framework. For the former we observe no phase transition but only a quick convergence to a $\sigma$-almost-consensus in $\mathcal{O}(1)$ rounds, with high probability (Proposition 6.1). The latter, instead, exhibits again a sharp transition, as that described before for $k$-MAJORITY, on the critical value $p^{\star}=\frac{1}{2}$ (Proposition 6.2). We also discuss, in Proposition 6.3, differences and similarities between this dynamics and $k$-MAJORITY for large values of $k$.

From a high level perspective, our results show that adding a bias to $k$-MAJORITY affects the dynamics in a non-trivial way. In particular, the arise of a metastable phase makes the framework suitable to design distributed algorithms to recover planted partitions in networks CNS19, BCPR19, SS19. In this direction, we discuss potential applications of the mathematical framework presented in this paper in Section 7 .

Most of the proofs of the Sections 4 to 6 are postponed to Sections 8 to 10 , respectively.

\section{Related Work}

Dynamics for consensus Simple mathematical model of interaction between nodes in a network have been studied since the first half of the 20th century in statistical mechanics, e.g., to model interacting particle systems or ferromagnetism phenomena [Lig12]. The simplest dynamics of interaction between the nodes involve local majority-based changes of states, e.g., as in the voter model [HL ${ }^{+} 75$, DW83] or in the majority dynamics [KR03]. A substantial line of research has been devoted to study the use of such simple dynamics as lightweight distributed algorithms to solve complex tasks, mirroring the behavior of complex systems from which they take inspiration. Here we are interested in discussing some of the contributions among the large body of work on dynamics for consensus. The reader is deferred to [BCN20] for a more detailed survey on the topic. All dynamics taken into consideration share a common communication model, where nodes can pull information from some fixed number of neighbors before updating their state.

As discussed in Section 1, vOTER is the first - and arguably the simplest - dynamics considered for consensus [HP01. The 2-CHOICES dynamics is a variation of vOTER in which nodes sample two random neighbors and update their states to the majority among two, breaking ties toward their own states. The dynamics has been studied with opinions on $d$-regular and expander graphs [CER14, proving that, given a sufficient initial unbalance between the two opinions, a consensus on the initial majority is reached within a polylogarithmic number of rounds, with high probability. Such results have been later improved in [CER ${ }^{+}$], relaxing the assumptions on graph's expansion, and generalized to the case of multiple opinions [CRRS17, EFK ${ }^{+} 17$ ]. More recently, the 2-CHOICES dynamics has been analyzed on networks with a core-periphery structure [CNNS18, where, depending on the initialization, it exhibits a phase transition phenomenon.

In the 3-MAJORITY dynamics, nodes update their color to that of the majority among the state of 3 randomly sampled neighbors. On the complete graph and with $h$ possible opinions, the process converges to a plurality consensus in $\mathcal{O}(\min \{h, \sqrt[3]{n / \log n}\} \cdot \log n)$ with high probability, if the initial unbalance between the plurality color and the second one is large enough $\left[\mathrm{BCN}^{+} 17\right]$. In [ $\left.\mathrm{BCE}^{+} 17\right]$ unconditional lower and upper bounds for 2-CHOICES and 3MAJORITY on the complete graph are provided, whenever the number of initial colors is large. 
The scenario in which an adversary can modify some of the $h$ opinions, again for 2-CHOICES and/or 3-MAJORITY, is considered in $\mathrm{BCN}^{+} 16, \mathrm{DGM}^{+} 11, \mathrm{GL18}$, with the best result proving convergence to a valid consensus in $\mathcal{O}(h \log n)$ rounds, with high probability, even if the adversary can control $o(\sqrt{n})$ nodes. The 3-MAJORITY dynamics has been recently analyzed also on non complete topologies [KR19. The authors consider a random initialization in which every node is red with probability $1 / 2+\delta$ and blue otherwise and graphs with minimum degree $d=\Omega\left(n^{1 / \log \log n}\right)$. Their result implies, e.g., a consensus on state red in $\mathcal{O}(\log \log n)$ rounds, w.h.p., if $\delta=\Omega(1 / \log \log n)$.

To the best of our knowledge, $k$-MAJORITY has not been extensively studied for generic $k$. Among the few works that consider it, in ABF15, AD15 such a dynamics is analyzed for $k \geq 5$ on preferential attachment graphs, on the configuration model, and on Erdős-Rényi graphs. In both works the authors show that, given an initial unbalance toward one of the two possible colors, the process converges to the initial majority within $\mathcal{O}(\log \log n)$ steps, with high probability. In [SS20] a new model is proposed, which contains majority rules as special cases. In particular, for $k$-MAJORITY with odd $k$ and in a binary state setting, the convergence time on expander graphs is proved to be $\mathcal{O}(\log n / \log h)$ rounds for $k=o(n / \log n)$.

In the DETERMINISTIC MAJORITY, every node updates its state according to the majority state of its neighborhood as a whole, loosing the random interaction, which is a fundamental feature of the dynamics previously discussed. This deterministic protocol has been extensively studied in the literature; we mention, for example, its analysis on expander graphs [MNT14, Zeh20], random regular graphs [GZ18], and Erdös-Rnyi random graphs [BCO ${ }^{+} 16$, Zeh20].

Biased communication models in opinion dynamics A different perspective coming from other disciplines, such as economics and sociology, is that of considering interaction models between nodes of a network as models of opinion diffusion. The main models, e.g., DeGroot DeG74] and Friedkin-Johnsen [FJ90, are based on averaging dynamics, i.e., nodes move toward the average opinion seen in their neighborhoods. Nevertheless, also the dynamics previously discussed can be framed in the modeling of opinion dynamics (see, e.g., ABB19, AFG18, MBCD19, MT17, SLST17).

Some opinion dynamics have been considered with biased communication models, specially in asynchronous case. The binary DETERMINISTIC MAJORITY dynamics has been considered in a setting where nodes have a fixed private opinion and, when active, announce a public opinion as the majority opinion in their neighborhood, but ties are broken toward their private belief. Such a process has been proved to converge to the initial private majority whenever the graphs are sufficiently sparse and expansive [FILW14] or preferential attachment trees [BIMW20]. The binary DETERMINISTIC MAJORITY dynamics, as well as the binary vOTER dynamics, have also been analyzed in asynchronous models presenting different forms of bias [MMR16, $\left.\mathrm{ABC}^{+} 20\right]$. In [MMR16], if the network prefers, say, opinion $a$ instead of $b$, every node holding opinion $b$ updates more frequently than the others; this particular feature is modeled by allowing nodes in state $b$ to revise their opinion at all points of a Poisson process with rate $q_{b}>q_{a}$. In $\left[\mathrm{ABC}^{+} 20\right.$. the bias is defined toward one of the two possible opinions: nodes have a fixed probability $\alpha$ of updating their state to such an opinion, independently of the dynamics.

A biased version of VOTER has also been studied in a synchronous model in [BGKM16]: the nodes, after selecting a random neighbor, have a probability of copying its state that depends on the state itself. 


\section{Notation and Computational Model}

Let $G=(V, E)$ be a simple graph with $V=\{1, \ldots, n\}$. For each node $u \in V$, let $N_{u}:=\{v \in$ $V:(u, v) \in E\}$ be the neighborhood of $u$. In the following, we focus only on sufficiently dense graphs, i.e., graphs where every node $u \in V$ has degree $\delta_{u}:=\left|N_{u}\right|=\omega(\log n)$ ! We use the Bachmann-Landau notation (i.e., $\omega, \Omega, \Theta, \mathcal{O}, o$ ) to describe the limiting behavior of functions depending on $n$. We denote the volume of a set of nodes $T \subseteq V$ as $\operatorname{vol}(T):=\sum_{v \in T} \delta_{v}$.

We consider a process on $G$ that evolves in discrete, synchronous rounds ${ }^{2}$ where, in every round $t \in \mathbb{N}_{0}$, every node $u \in V$ has a binary state $x_{u}^{(t)} \in\{\mathcal{R}, \mathcal{B}\}$ that can change over time according to a function of the states of its neighbors; we denote the configuration of the system at round $t$, i.e., the vector of states of the nodes of $G$, as $\mathbf{x}^{(t)} \in\{\mathcal{R}, \mathcal{B}\}^{n}$; we define $R^{(t)}:=\left\{u \in V: x_{u}^{(t)}=\mathcal{R}\right\}$ and $B^{(t)}:=\left\{u \in V: x_{u}^{(t)}=\mathcal{B}\right\}$.

We let the nodes of the network run the $(k, p, \sigma)$-MAJORITY dynamics (formally introduced in Definition 3.1), a slight modification of the well-known $k$-MAJORITY dynamics where the communication between nodes is biased (through a parameter $p$ ) toward one of the two possible states, $\sigma$. Recall that in the $k$-MAJORITY dynamics, in each round, every node samples $k$ neighbors uniformly at random and with replacement; then it updates its state to the state held in the previous round by the majority of the neighbors in the sample; ties are broken uniformly at random. Differently, in the $(k, p, \sigma)$-MAJORITY dynamics, whenever node $u$ samples a neighbor $v$, the state of $v$ seen by $u$ could be altered, i.e., $v$ is seen in state $\sigma$, with probability $p$, regardless of its actual state.

We denote by $\mathbf{P}=\mathbf{P}^{(n)}$ the law of the dynamics on $G$. We usually drop the dependence on $n$ when it is clear from the context. We use the notation $\mathbb{1}_{\{\mathcal{A}\}}$ for the indicator variable of the event $\mathcal{A}$, i.e., $\mathbb{1}_{\{\mathcal{A}\}}=1$ if $\mathcal{A}$ holds and $\mathbb{1}_{\{\mathcal{A}\}}=0$ otherwise.

Formally, $(k, p, \sigma)$-MAJORITY can be described as follows. Let $p \in[0,1]$ be the parameter that models the bias toward some state $\sigma \in\{\mathcal{R}, \mathcal{B}\}$; let $k \in \mathbb{N}$ be the size of the sampling. For each round $t$, let $S_{u}^{(t)}$ be the multiset ${ }^{3}$ of neighbors sampled by node $u$ in round $t$. For each sampled node $v \in S_{u}^{(t)}$, we call $\bar{x}_{v}^{(t)}(u)$ the state in which node $u$ sees $v$ after the effect of the bias, i.e., $\bar{x}_{v}^{(t)}(u)=\sigma$ with probability $p$, independently of the state of $v$, and $\bar{x}_{v}^{(t)}(u)=x_{v}^{(t)}$ otherwise; formally

$$
\mathbf{P}\left(\bar{x}_{v}^{(t)}(u)=\sigma \mid \mathbf{x}^{(t)}=\overline{\mathbf{x}}\right)=\mathbb{1}_{\left\{x_{v}^{(t)}=\sigma\right\}}+p \cdot \mathbb{1}_{\left\{x_{v}^{(t)} \neq \sigma\right\}} .
$$

We define $\bar{R}_{u}^{(t)}:=\left\{v \in S_{u}^{(t)}: \bar{x}_{v}^{(t)}(u)=\mathcal{R}\right\}$ and $\bar{B}_{u}^{(t)}:=\left\{v \in S_{u}^{(t)}: \bar{x}_{v}^{(t)}(u)=\mathcal{B}\right\}$ as the sets that node $u$ sees respectively in state $\mathcal{R}$ and in state $\mathcal{B}$ after the effect of the bias $p$.

Definition $3.1((k, p, \sigma)$-MAJORITY dynamics). Let $p \in[0,1], k \in \mathbb{N}$, and $\sigma \in\{\mathcal{R}, \mathcal{B}\}$. Starting from an initial configuration $\mathbf{x}^{(0)}$, at each round $t$ every node $u \in V$ decides its state for the next round as

$$
x_{u}^{(t+1)}= \begin{cases}\mathcal{R} & \text { if }\left|\bar{R}_{u}^{(t)}\right|>\left|\bar{B}_{u}^{(t)}\right|, \\ \mathcal{R} \text { or } \mathcal{B} \text { with probability } 1 / 2 & \text { if }\left|\bar{R}_{u}^{(t)}\right|=\left|\bar{B}_{u}^{(t)}\right|, \\ \mathcal{B} & \text { if }\left|\bar{R}_{u}^{(t)}\right|<\left|\bar{B}_{u}^{(t)}\right|\end{cases}
$$

Note that the $(k, p, \sigma)$-MAJORITY dynamics is a Markov Chain, since the configuration $\mathbf{x}^{(t)}$ in a round $t>0$ depends only on the configuration $\mathbf{x}^{(t-1)}$ at the previous round. Moreover,

\footnotetext{
${ }^{1}$ We refer to such graphs as "dense" in order to highlight the lower bound on the degree of the nodes that is necessary for our proof technique.

${ }^{2}$ Equivalently, nodes have access to a shared clock.

${ }^{3}$ Recall that the sampling is with replacement.
} 
when $p>0$, it has a single absorbing state, in which $x_{u}^{(t)}=\sigma$ for every $u \in V$, i.e., eventually all nodes reach a consensus on state $\sigma$. Notice also that our dynamics is monotone and hence the time needed to reach the $\sigma$-consensus decreases if we increase the number of vertices in state $\sigma$ in the current configuration. For this reason, in Section 5.1 we consider the scenario in which all nodes are initially in the state opposite to $\sigma$. Without loss of generality, in what follows we let $x_{u}^{(0)}=\mathcal{R}$ for every node $u \in V$ and let the nodes in $G$ run the $(k, p, \mathcal{B})$-MAJORITY dynamics. Later, in Section 5.2 , we analyze the general scenario in which nodes are initially in state $\mathcal{R}$, independently, with probability $q$. We examine these scenarios in order to understand the effect of the bias and of the initial unbalance toward state $\mathcal{R}$ on the process. In particular, we study the time needed by the process to reach a $\mathcal{B}$-almost-consensus, i.e., a configuration in which most of the nodes are in state $\mathcal{B}$. More precisely, we give the following definition.

Definition 3.2 ( $\sigma$-almost-consensus). Let $\sigma \in\{\mathcal{R}, \mathcal{B}\}$ and let $\Sigma^{(t)}:=\left\{u \in V: x_{u}^{(t)}=\sigma\right\}$, for each round $t$. For any constant $\gamma \in(0,1)$, consider the stopping time

$$
\tau_{\gamma}:=\inf \left\{\begin{array}{l|l}
t \geq 0 & \frac{\operatorname{vol}\left(\Sigma^{(t)}\right)}{\operatorname{vol}(V)}>1-\gamma
\end{array}\right\} .
$$

We say that a process on $G$ reaches a $\sigma$-almost-consensus within $\mathcal{O}(f(n))$ rounds if for any constant $\gamma>0$ there exists some constant $c=c(\gamma)$ such that

$$
\mathbf{P}\left(\tau_{\gamma} \leq c f(n)\right)=1-o(1) .
$$

In the following sections, we say that an event $\mathcal{E}_{n}$ holds with high probability (w.h.p., in short) if $\mathbf{P}\left(\mathcal{E}_{n}\right)=1-o(1)$. In this sense, our results only hold for large $n$. We also use the notation $\operatorname{Bin}(n, p)$ to indicate a random variable sampled by the Binomial distribution of parameters $n$ (number of trials) and $p$ (probability of success).

\section{A Toy Model: Infinite Trees}

We start the analysis with a "toy model", i.e., a directed infinite tree $\mathcal{T}=(V, E)$ with edges oriented toward the children. We call $v_{0} \in V$ the root of $\mathcal{T}$. For each node $u \in V$ we define the binary state $x_{u}^{(t)} \in\{\mathcal{R}, \mathcal{B}\}$ that can change over time as described in Definition 3.1, but with the only difference that $S_{u}^{(t)}$ will be a set of $k$ children sampled by node $u$ at round $t$, without replacement; this difference in the dynamics will allow us to analyze the behavior of the processes using the same techniques. Hence, fixed the bias $p \in[0,1]$, for each $v \in S_{u}^{(t)}$ we can define the binary random variable $\bar{x}_{v}^{(t)}(u)$ and at each round $t$ we can construct the sets $R^{(t)}$ and $B^{(t)}$, as discussed in Section 3 .

Suppose that $k$ is odd. We study the evolution of the probability of the event " $v_{0} \in R^{(t)}$ ", i.e., the root of $\mathcal{T}$ is in state $\mathcal{R}$ at round $t$. The result in the forthcoming Theorem 4.3 is based on the analysis of the function $F_{p, k}$, described by the following definition, that represents the evolution of the event under analysis.

Definition 4.1 (Function $F_{p, k}$ ). Let $h \in \mathbb{N}$ and let $k:=2 h+1$. Let $p \in[0,1]$. We define the function $F_{p, k}:[0,1] \rightarrow[0,1]$ as

$$
F_{p, k}(x):=\mathbf{P}\left(\operatorname{Bin}(k,(1-p) x) \geq \frac{k+1}{2}\right) .
$$

In particular, we will use the following facts about $F_{p, k}$, which are proved in Section 8 and depicted in Fig. 1. 
Lemma 4.2. For every finite odd $k \geq 3$, there exists $p_{k}^{\star} \in\left[\frac{1}{9}, \frac{1}{2}\right)$ such that:

- if $p<p_{k}^{\star}$, there exist $\varphi_{p, k}^{-}, \varphi_{p, k}^{+} \in\left(\frac{1}{2(1-p)}, 1\right]$ with $\varphi_{p, k}^{-}<\varphi_{p, k}^{+}$such that $F_{p, k}(x)=x$ has solutions $0, \varphi_{p, k}^{-}$, and $\varphi_{p, k}^{+}$;

- if $p=p_{k}^{\star}$, there exists $\varphi_{p, k} \in\left(\frac{1}{2(1-p)}, 1\right]$ such that $F_{p, k}(x)=x$ has solutions 0 and $\varphi_{p, k}$;

- if $p>p_{k}^{\star}$, then $F_{p, k}(x)=x$ has 0 as unique solution.

Moreover the sequence $\left\{p_{k}^{\star}\right\}_{k}$ is increasing.

Note that it is not possible to give a closed formula of $p_{k}^{\star}$ for generic $k$ because it is the root of a polynomial of degree $k$; numerical approximations can be computed for any given $k$. However, as proved in Lemma $4.2, p_{k}^{\star}$ monotonically increases with $k$, starting from $\frac{1}{9}$ (for $k=3$ ) and up to $\frac{1}{2}$ (its limit value as $k \rightarrow \infty$, as proved in Lemma 8.3.
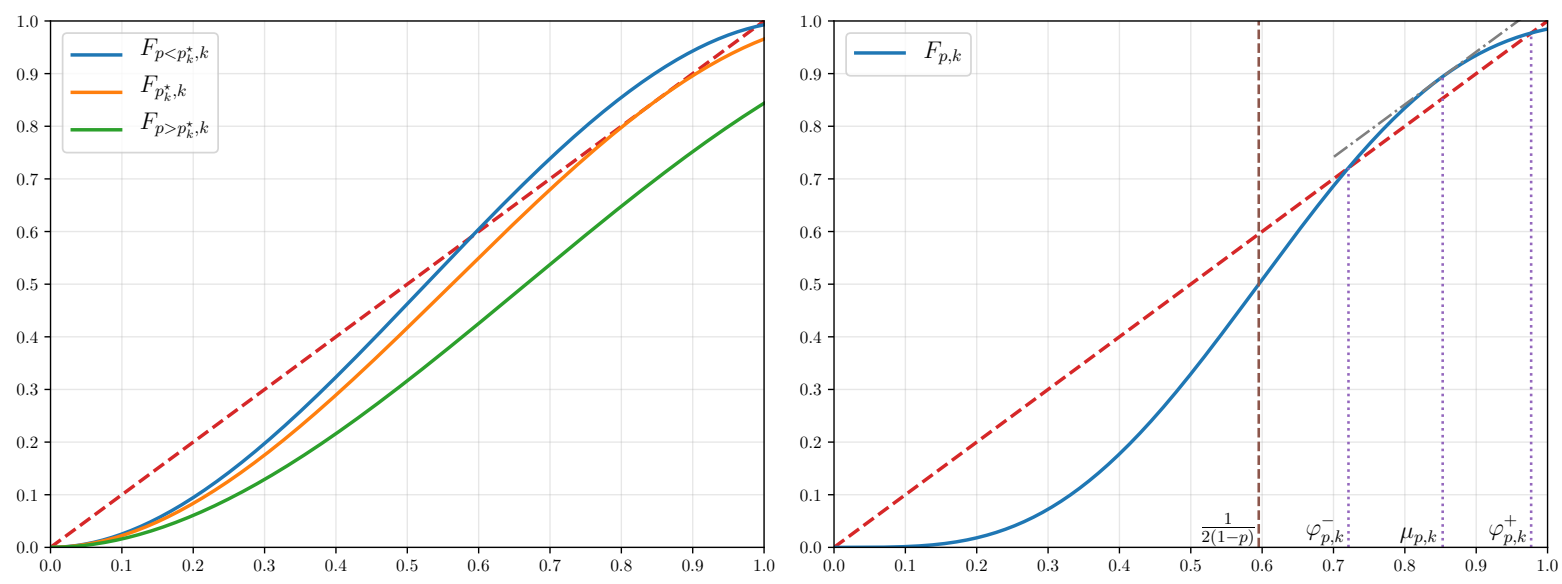

Figure 1: Qualitative plots of the function $F_{p, k}(x)$. On the left, for $k=3$, three different values of $p$ are shown, namely $p=\frac{1}{20}<p_{k}^{\star}, p=\frac{1}{9}=p_{k}^{\star}$, and $p=\frac{1}{4}>p_{k}^{\star}$. On the right, for $k=7$ and $p=0.16<p_{k}^{\star}$, the points of interest of the function are shown, namely the inflection point $\frac{1}{2(1-p)}$, the fixed points $\varphi_{p, k}^{-}$and $\varphi_{p, k}^{+}$, and the unique point $\mu_{p, k}>\varphi_{p, k}^{-}$for which $F_{p, k}^{\prime}\left(\mu_{p, k}\right)=1$.

We now prove the following theorem, which will be a guideline for the whole paper.

Theorem 4.3. Consider the $(k, p, \mathcal{B})$-MAJORITY dynamics on $\mathcal{T}$, where at round $t=0$ each vertex of the tree is in state $\mathcal{R}$ with probability $q$ or in state $\mathcal{B}$ with probability $1-q$, independently of the others. Then,

$$
\lim _{t \rightarrow \infty} \mathbf{P}\left(v_{0} \in R^{(t)}\right)= \begin{cases}\varphi_{p, k}^{+} & \text {if } p<p_{k}^{\star} \text { and } q>\varphi_{p, k}^{-} \\ 0 & \text { if } p<p_{k}^{\star} \text { and } q<\varphi_{p, k}^{-} \\ 0 & \text { if } p>p_{k}^{\star} .\end{cases}
$$

Proof. Define $q_{t}:=\mathbf{P}\left(v_{0} \in R^{(t)}\right)$ and observe that, since we are working on an infinite tree, we have $\mathbf{P}\left(v_{0} \in R^{(t)}\right)=\mathbf{P}\left(v \in R^{(t)}\right)$ for all $v \in V$ and for all $t \in \mathbb{N}_{0}$. Note also that, for any siblings $v, w \in V$, the events " $v \in R^{(t)}$ " and " $w \in R^{(t)}$ " are independent at any round $t \in \mathbb{N}_{0}$. Hence the random variables in the family $\left\{\mathbb{1}_{\left\{v \in R^{(t)}\right\}}: v \in V\right\}$ are i.i.d. and $\mathbf{P}\left(\mathbb{1}_{\left\{v \in R^{(t)}\right\}}=1\right)=q_{t}$. 
Let us now compute $q_{t+1}=\mathbf{P}\left(v_{0} \in R^{(t+1)}\right)$. We have:

$$
q_{t+1}=\mathbf{P}\left(\sum_{w \in S_{v_{0}}^{(t)}} \mathbb{1}_{\left\{w \in R^{(t)}\right\}} \mathbb{1}_{\left\{\bar{x}_{w}^{(t)}\left(v_{0}\right)=x_{w}^{(t)}\right\}} \geq \frac{k+1}{2}\right) .
$$

Note that the random variables $\left\{\mathbb{1}_{\left\{\bar{x}_{w}^{(t)}\left(v_{0}\right)=x_{w}^{(t)}\right\}} \mid w \in S_{v_{0}}^{(t)}\right\}$ are i.i.d. Bernoulli random variables of parameter $1-p$. Moreover the family of random variables $\left\{\mathbb{1}_{\left\{\bar{x}_{w}^{(t)}\left(v_{0}\right)=x_{w}^{(t)}\right\}} \mid w \in S_{v_{0}}^{(t)}\right\}$ is independent of the family $\left\{\mathbb{1}_{\left\{w \in R^{(t)}\right\}} \mid w \in S_{v_{0}}^{(t)}\right\}$. Then $\left\{\mathbb{1}_{\left\{w \in R^{(t)}\right\}} \mathbb{1}_{\left\{\bar{x}_{w}^{(t)}\left(v_{0}\right)=x_{w}^{(t)}\right\}} \mid w \in S_{v_{0}}^{(t)}\right\}$ is a family of i.i.d. Bernoulli random variables of parameter $(1-p) q_{t}$ and hence

$$
\sum_{w \in S_{v_{0}}^{(t)}} \mathbb{1}_{\left\{w \in R^{(t)}\right\}} \mathbb{1}_{\left\{\bar{x}_{w}^{(t)}\left(v_{0}\right)=x_{w}^{(t)}\right\}}=\operatorname{Bin}\left(\left|S_{v_{0}}^{(t)}\right|,(1-p) q_{t}\right)=\operatorname{Bin}\left(k,(1-p) q_{t}\right) .
$$

Thus, for every $t \geq 0$, we can write

$$
q_{t+1}=\mathbf{P}\left(\operatorname{Bin}\left(k,(1-p) q_{t}\right) \geq \frac{k+1}{2}\right) .
$$

By Definition 4.1, the sequence described in Eq. (3) can be rewritten as

$$
q_{t+1}= \begin{cases}F_{p, k}(q) & \text { if } t=0 \\ F_{p, k}\left(q_{t}\right) & \text { if } t \geq 1\end{cases}
$$

Hence, the limit behavior in Eq. (1) for the sequence $\left(q_{t}\right)_{t}$ follows from Lemma 4.2 .

\section{Phase Transitions}

In this section we exploit the results in Section 4 to prove that $(k, p, \sigma)$-MAJORITY behaves on dense graphs similarly to what we observed in the previous section on infinite trees.

We start by setting the ground for Theorem 5.1. introducing the required notation. For every node $u \in V$ and for every round $t$ we define the fraction of neighbors of $u$ in state $\mathcal{R}$ as $\phi_{u}^{(t)}:=\frac{\left|R_{u}^{(t)}\right|}{\delta_{u}}$. Similarly we let $\phi_{\max }^{(t)}:=\max _{u \in V} \phi_{u}^{(t)}$ denote the maximum fraction of neighbors in state $\mathcal{R}$ at round $t$ over the nodes.

Given any configuration $\mathbf{x}^{(t)}=\overline{\mathbf{x}}$, we have that, for every $u \in V$, the expected fraction of neighbors of $u$ in state $\mathcal{R}$ at round $t+1$ is

$$
\mathbf{E}\left[\phi_{u}^{(t+1)} \mid \mathbf{x}^{(t)}=\overline{\mathbf{x}}\right]=\frac{1}{\delta_{u}} \sum_{v \in N_{u}} \mathbf{P}\left(\left|\bar{R}_{v}^{(t)}\right| \geq \frac{k+1}{2} \mid \mathbf{x}^{(t)}=\overline{\mathbf{x}}\right)=\frac{1}{\delta_{u}} \sum_{v \in N_{u}} F_{p, k}\left(\phi_{v}^{(t)}\right) .
$$

Note that in the last equality we applied Definition 4.1, and used the fact that, given $\mathbf{x}^{(t)}$, the random variable $\left|\bar{R}_{v}^{(t)}\right|=\operatorname{Bin}\left(k,(1-p) \phi_{v}^{(t)}\right)$, for every $v \in V$.

\subsection{On the Bias in the Communication $p$}

The main result of this work shows the existence of a critical value $p_{k}^{\star}$ (see Lemma 4.2) for the bias $p$ when the nodes of a dense graph execute $(k, p, \mathcal{B})$-MAJORITY starting from an initial configuration where all nodes are in state $\mathcal{R}$. Roughly speaking, on the one hand Theorem 5.1 
states that, if the bias is smaller than $p_{k}^{\star}$, a superpolynomial number of rounds is needed to reach a $\mathcal{B}$-almost-consensus: we will show that the system will remain trapped in a metastable phase, in which the volume of nodes in state $\mathcal{R}$ is some constant fraction of the total, for every polynomial number of rounds. On the other hand, if the bias is larger than $p_{k}^{\star}$, a constant number of rounds suffices to reach a $\mathcal{B}$-almost-consensus.

Theorem 5.1. Consider a sequence of graphs $\left(G_{n}\right)_{n \in \mathbb{N}}$ such that $\min _{v \in V} \delta_{v}=\omega(\log n)$. For every fixed $n$, consider the $(k, p, \mathcal{B})$-MAJORITY dynamics, with $p \in[0,1]$ and $k \geq 3$, starting from the initial configuration $\mathbf{x}^{(0)}$ where $x_{u}^{(0)}=\mathcal{R}$ for every $u \in V$. If $\left|p-p_{k}^{\star}\right|>c$ for some constant $c>0$, then:

1. Slow convergence: if $p<p_{k}^{\star}$, then for every constant $\gamma>0$ there exists a $T=T(\gamma)$ s.t.

$$
\mathbf{P}\left(\forall t \in\left[T, n^{K}\right], \frac{\operatorname{vol}\left(R^{(t)}\right)}{\operatorname{vol}(V)} \in\left[\varphi_{p, k}^{+}-\gamma, \varphi_{p, k}^{+}+\gamma\right]\right)=1-o(1), \quad \forall K>0,
$$

where $\varphi_{p, k}^{+}$is the largest fixed point of $F_{p, k}$ (see Lemma 4.2).

2. Fast convergence: if $p>p_{k}^{\star}$, then for every constant $\gamma>0$ there exists a $T=T(\gamma)$ s.t.

$$
\mathbf{P}\left(\exists t<T \text { s.t. } \frac{\operatorname{vol}\left(B^{(t)}\right)}{\operatorname{vol}(V)}>1-\gamma\right)=1-o(1) .
$$

In the rest of the section we assume $k$ is odd and we prove the theorem for this case only. We later show in Proposition 5.11 that this assumption is not necessary. In fact, the behavior of $(k, p, \mathcal{B})$-MAJORITY is equivalent to that of $(k+1, p, \mathcal{B})$-MAJORITY for every odd $k$.

We split the proof into two parts. Theorem 5.1 then follows from Corollaries 5.3 and 5.6 .

Slow convergence The proof for the slow convergence regime directly exploits the connection with the infinite tree model described in Section 4. In Proposition 5.2 we show that for every round $t \in \operatorname{poly}(n)$ all the nodes in $G$ have a fraction of neighbors in state $\mathcal{R}$ which is asymptotically equal to $q_{t}$, i.e., the probability that the root $v_{0}$ of the infinite tree $\mathcal{T}$ is in state $\mathcal{R}$ at round $t$.

Proposition 5.2. Consider $p=p_{k}^{\star}-c$, for some $c>0$, so that the quantity $\varphi_{p, k}^{-}$in Lemma 4.2 is well defined. Consider the $(k, p, \mathcal{B})$-MAJORITY starting from the initial configuration in which each vertex is $\mathcal{R}$. Then, for all $\gamma>0$,

$$
\mathbf{P}\left(\forall t \leq n^{K}, \forall v \in V, \phi_{v}^{(t)} \in\left[q_{t}-\gamma, q_{t}+\gamma\right]\right)=1-o(1), \quad \forall K>0 .
$$

where the sequence $\left(q_{t}\right)_{t \geq 0}$ is defined recursively as in Section 4, by choosing $q=1$.

The result in Proposition 5.2 is discussed and proved for a more general class of initial conditions (Proposition 9.1) in Section 9. The core of the proof lies in the contraction property of $F_{p, k}$. The latter is stated rigorously in Lemma 8.2 , where we show that $F_{p, k}^{\prime}<1$ in that interval $\left[\varphi_{p, k}^{+}, 1\right]$. The proof of Proposition 5.2 amounts to show that this fact is sufficient to ensure a bilateral concentration. Moreover, given the density assumption, the concentration can be pushed to hold uniformly in the vertices at any polynomial round.

By Proposition 5.2 the fraction of $\mathcal{R}$ neighbors of a node concentrates around $q_{t}$, i.e., the probability that the root of the infinite tree is in state $\mathcal{R}$ at round $t$. Then, we use Proposition 5.2 to show that a metastable phase - in which the fraction of volume in state $\mathcal{R}$ is approximately $\varphi_{p, k}^{+}$-is attained in constant time. Moreover, an additional superpolynomial number of rounds is necessary to reach a $\mathcal{B}$-almost-consensus. 
Corollary 5.3. Assume $p=p_{k}^{\star}-c$ for some constant $c>0$ and that the initial configuration is such that all nodes are in state $\mathcal{R}$. Then for all constant $\gamma>0$ there exists a sufficiently large $T=T(\gamma)$ such that

$$
\mathbf{P}\left(\forall t \in\left[T, n^{K}\right], \frac{\operatorname{vol}\left(R^{(t)}\right)}{\operatorname{vol}(V)} \in\left[\varphi_{p, k}^{+}-\gamma, \varphi_{p, k}^{+}+\gamma\right]\right)=1-o(1), \quad \forall K>0 .
$$

Proof. Since the sequence $\left(q_{t}\right)_{t \geq 0}$ does not depend on $n$, Proposition 5.2 implies that

$$
\mathbf{P}\left(\forall t \in\left[T, n^{K}\right], \forall v \in V, \phi_{v}^{(t)} \in\left[\varphi_{p, k}^{+}-\gamma, \varphi_{p, k}^{+}+\gamma\right]\right)=1-o(1) .
$$

At this point it is sufficient to rewrite the volume of nodes in state $\mathcal{R}$ as follows

$$
\operatorname{vol}\left(R^{(t)}\right)=\sum_{u \in R^{(t)}} \delta_{u}=\sum_{u \in V}\left(\delta_{u}-\left|B_{u}^{(t)}\right|\right)=\sum_{u \in V} \delta_{u} \phi_{u}^{(t)}
$$

Then, by Proposition 5.2 , for all $K>0,\left(\varphi_{p, k}^{+}-\gamma\right) \operatorname{vol}(V) \leq \operatorname{vol}\left(R^{(t)}\right) \leq\left(\varphi_{p, k}^{+}+\gamma\right) \operatorname{vol}(V)$, for all $v$ and all $t \in\left[T, n^{K}\right]$.

Fast convergence In the fast convergence regime, for every node of the graph, we can upper bound the expected fraction of neighbors in state $\mathcal{R}$. This fact is formalized in the following lemma, which is proved in Section 9.

Lemma 5.4. Let $p=p_{k}^{\star}+c$, for some $c>0$. There exists some $\varepsilon=\varepsilon(p, k)>0$ such that for every $u \in V$ it holds that

$$
\mathbf{E}\left[\phi_{u}^{(t+1)} \mid \mathbf{x}^{(t)}=\overline{\mathbf{x}}\right] \leq(1-\varepsilon) \phi_{\max }^{(t)} .
$$

Lemma 5.4 essentially tells us that the fraction of neighbors of $u$ in state $\mathcal{R}$, maximized over $u \in V$, is a supermartingale. We will use this fact to prove the following proposition.

Proposition 5.5. Fix $p=p_{k}^{\star}+c$, for some $c>0$. For all $\gamma>0$ there exists some $T=T(\gamma)$ such that

$$
\mathbf{P}\left(\exists t \leq T \text { s.t. } \phi_{\max }^{(t)} \leq \gamma\right)=1-o(1) .
$$

Proof. For any $u \in V$, by multiplying both sides of Eq. (6) by $\delta_{u}$ we get that, for all $\gamma>0$,

$$
\mathbf{E}\left[\left|R_{u}^{(t+1)}\right| \mid \mathbf{x}^{(t)}=\overline{\mathbf{x}}\right] \leq(1-\varepsilon) \phi_{\max }^{(t)} \delta_{u} \leq(1-\varepsilon) \max \left\{\phi_{\max }^{(t)}, \gamma\right\} \delta_{u} .
$$

We aim at bounding the quantity $\phi_{u}^{(t+1)}$, namely

$$
\begin{aligned}
\mathbf{P}\left(\phi_{u}^{(t+1)}>\left(1-\varepsilon^{2}\right) \max \left\{\phi_{\max }^{(t)}, \gamma\right\} \mid\right. & \left.\mathbf{x}^{(t)}=\overline{\mathbf{x}}\right) \\
=\mathbf{P}\left(\left|R_{u}^{(t+1)}\right|\right. & \left.>\left(1-\varepsilon^{2}\right) \max \left\{\phi_{\max }^{(t)}, \gamma\right\} \delta_{u} \mid \mathbf{x}^{(t)}=\overline{\mathbf{x}}\right) \\
= & \mathbf{P}\left(\left|R_{u}^{(t+1)}\right|>(1+\varepsilon)(1-\varepsilon) \max \left\{\phi_{\max }^{(t)}, \gamma\right\} \delta_{u} \mid \mathbf{x}^{(t)}=\overline{\mathbf{x}}\right) .
\end{aligned}
$$

Note that, given the configuration at round $t,\left|R_{u}^{(t+1)}\right|$ is a Binomial random variable and, thus, by applying a multiplicative form of the Chernoff Bound [DP09, Exercise 1.1], we get

$$
\mathbf{P}\left(\phi_{u}^{(t+1)}>\left(1-\varepsilon^{2}\right) \max \left\{\phi_{\max }^{(t)}, \gamma\right\} \mid \mathbf{x}^{(t)}=\overline{\mathbf{x}}\right) \leq \exp \left\{-\frac{\varepsilon^{2}(1-\varepsilon)}{3} \max \left\{\phi_{\max }^{(t)}, \gamma\right\} \delta_{u}\right\} .
$$


Since $\delta_{u}=\omega(\log n)$ by hypothesis and $\max \left\{\gamma, \phi_{\max }^{(t)}\right\}>\gamma$ regardless of $\phi_{\max }^{(t)}$, by taking a union bound over $u \in V$ and integrating over the conditioning we get

$$
\mathbf{P}\left(\phi_{\max }^{(t+1)}>\left(1-\varepsilon^{2}\right) \max \left\{\gamma, \phi_{\max }^{(t)}\right\}\right) \leq n \cdot e^{-\omega(\log n)} .
$$

Let us define the event $\mathcal{F}:=\left\{\forall t<T, \phi_{\max }^{(t+1)} \leq\left(1-\varepsilon^{2}\right) \max \left\{\gamma, \phi_{\max }^{(t)}\right\}\right\}$. By taking a union bound of Eq. (8) with respect to $t<T$ we have $\mathbf{P}(\mathcal{F}) \geq 1-T \cdot n \cdot e^{-\omega(\log n)}$. Called $\mathcal{E}:=$ $\left\{\exists t \leq T\right.$ s.t. $\left.\phi_{\max }^{(t)} \leq \gamma\right\} \supset \mathcal{F}$, we get the thesis.

Reasoning as in Corollary 5.3 it is immediate to deduce from Proposition 5.5 a statement about convergence to $\mathcal{B}$-almost-consensus.

Corollary 5.6. Assume $p=p_{k}^{\star}+c$ for some constant $c>0$. Then, for all $\gamma>0$ there exists a sufficiently large $T=T(\gamma)$ for which

$$
\mathbf{P}\left(\exists t<T \text { s.t. } \frac{\operatorname{vol}\left(B^{(t)}\right)}{\operatorname{vol}(V)}>1-\gamma\right)=1-o(1) .
$$

\subsection{On the Initial Unbalance $q$}

In this section we focus on the scenario in which, for any fixed odd $k$, we consider a value of the bias $p=p_{k}^{\star}-c$ for some constant $c>0$. We have seen in Section 5.1 that the time needed to reach a $\mathcal{B}$-almost-consensus is superpolynomial in the size of the graph when all nodes are initially in state $\mathcal{R}$. The natural question is: how large should the fraction of $\mathcal{B}$ vertices be in the initial configuration in order to reach the $\mathcal{B}$-almost-consensus in a much shorter time scale? In particular, we consider an initial condition in which each vertex is $\mathcal{R}$ with probability $q \in[0,1]$, independently of the others. Recall that $\varphi_{p, k}^{-}, \varphi_{p, k}^{+}$are the fixed points of $F_{p, k}$ when $p<p_{k}^{\star}$ (see Lemma 4.2 . We show that, if $q<\varphi_{p, k}^{-}$, then a $\mathcal{B}$-almost-consensus is reached in finite time; otherwise, if $q<\varphi_{p, k}^{-}$, the process enters a metastable phase in which the fraction of volume in state $\mathcal{R}$ concentrates around $\varphi_{p, k}^{+}$.

Theorem 5.7. Consider a sequence of graphs $\left(G_{n}\right)_{n \in \mathbb{N}}$ such that $\min _{v \in V} \delta_{v}=\omega(\log n)$. For every fixed $n$ consider the $(k, p, \mathcal{B})$-MAJORITY dynamics with $p \in(0,1)$ and odd $k \geq 3$. Assume that the system starts from an initial configuration $\mathbf{x}^{(0)}$ where each vertex is $\mathcal{R}$ with probability $q \in[0,1]$, independently of the others. If $p=p_{k}^{\star}-c$ for some constant $c>0$, and $\left|q-\varphi_{p, k}^{-}\right|>\eta$ for some $\eta>0$, then:

1. Slow convergence: if $q>\varphi_{p, k}^{-}$, then for all constant $\gamma>0$ there exists a constant $T=T(\gamma)$ s.t.

$$
\mathbf{P}\left(\forall t \in\left[T, n^{K}\right], \frac{\operatorname{vol}\left(R^{(t)}\right)}{\operatorname{vol}(V)} \in\left[\varphi_{p, k}^{+}-\gamma, \varphi_{p, k}^{+}+\gamma\right]\right)=1-o(1), \quad \forall K>0 .
$$

2. Fast convergence: if $q<\varphi_{p, k}^{-}$, then for all constant $\gamma>0$ there exists a $T=T(\gamma)$ s.t.

$$
\mathbf{P}\left(\exists t<T \text { s.t. } \frac{\operatorname{vol}\left(B^{(t)}\right)}{\operatorname{vol}(V)}>1-\gamma\right)=1-o(1) .
$$


Slow convergence As far as it concerns the slow convergence regime, the proof is divided in two steps. We start with Proposition 9.1, an extension of Proposition 5.2 that considers every starting configuration with $q>\mu_{p, k}$ (where $\mu_{p, k}$ is the unique point in $\left[\varphi_{p, k}^{-}, 1\right]$ for which $F_{p, k}^{\prime}\left(\mu_{p, k}\right)=1$, see Fig. 1). For such initial configurations we can rely on the contraction property of $F_{p, k}$.

The second part of the proof closes the gap where $q \in\left(\varphi_{p, k}^{-}, \mu_{p, k}\right]$. More precisely, we prove the following proposition.

Proposition 5.8. Let $p=p_{k}^{\star}-c$, for some $c>0$ and consider the $(k, p, \mathcal{B})$-MAJORITY starting from the initial configuration in which each vertex is $\mathcal{R}$ independently of the others, with probability $q \in\left[\varphi_{p, k}^{-}+\eta, \mu_{p, k}\right)$ for some $\eta>0$. Then, there exists some $\tau_{p, k}=\tau_{p, k}(q)$ such that $q_{\tau_{p, k}}>\mu_{p, k}$. Moreover, for all $\gamma>0$

$$
\mathbf{P}\left(\forall t \leq \tau_{p, k}, \forall v \in V, \phi_{v}^{(t)} \in\left[q_{t}-\gamma, q_{t}+\gamma\right]\right)=1-e^{-\omega(\log n)} .
$$

Informally, Proposition 5.8 (whose proof is in Section 9.2 ) claims that within a finite number of rounds every node has at least a fraction $\mu_{p, k}$ of neighbors in state $\mathcal{R}$. Moreover, the fraction of neighbors in state $\mathcal{R}$ of every vertex coincides at the first order. Then, at that finite time, we can apply Proposition 9.1 and conclude the proof as in Corollary 5.3 .

Fast convergence For the fast convergence regime, the proof is similar to the proof in the second half of Section 5.1. Analogously to what has been done in Lemma 5.4, we first upper bound the expected fraction of neighbors in state $\mathcal{R}$, for each node of the graph.

Lemma 5.9. Consider any $\mathbf{x}^{(t)}$ such that $\phi_{\max }^{(t)} \leq \varphi_{p, k}^{-}-\eta$, for some $\eta>0$. Then, there exists some constant $\varepsilon^{\prime}=\varepsilon^{\prime}(p, k, \eta)>0$ such that

$$
\mathbf{E}\left[\phi_{u}^{(t+1)} \mid \mathbf{x}^{(t)}=\overline{\mathbf{x}}\right] \leq\left(1-\varepsilon^{\prime}\right) \phi_{\max }^{(t)} .
$$

The proof of Lemma 5.9 can be found in Section 9.2. Then we make use of the next proposition, whose proof follows the same flow as that one of Proposition 5.5, and the conclusion follows by the same argument used in Corollary 5.6.

Proposition 5.10. Let $p=p_{k}^{\star}-c$, for some $c>0$. Consider $(k, p, \mathcal{B})$-MAJORITY starting from the initial configuration in which each vertex is in state $\mathcal{R}$, independently of the others, with probability $q=\varphi_{p, k}^{-}-\gamma$ for some $\gamma>0$. Then, for all $\gamma>0$ there exists some $T=T(\gamma)$ such that

$$
\mathbf{P}\left(\exists t \leq T \text { s.t. } \phi_{\max }^{(t)} \leq \gamma\right)=1-o(1) .
$$

\subsection{On Even Values of the Sample Size $k$}

In the previous section we assumed samples of odd size $k$, thus avoiding potential ties. In this section, we close the gap showing the equivalence between the Markov chains $(2 h+1, p, \sigma)$ MAJORITY and $(2 h+2, p, \sigma)$-MAJORITY. The result in Proposition 5.11 is essentially a special case of [FN19, Appendix B]. However, it has been obtained independently and a simplified proof is reported for completeness in Section 9.3 .

Proposition 5.11. Let $G=(V, E)$ be a graph with binary state configuration $\mathbf{x}^{(t)}$ in round $t$. Consider the $(k, p, \sigma)$-MAJORITY dynamics, for some fixed $k \in \mathbb{N}, p \in[0,1]$, and $\sigma \in\{\mathcal{R}, \mathcal{B}\}$. Pick a node $u \in V$ and define the event $\mathcal{E}_{k}$ : "Node $u$ does not update its state to $\sigma$ at round $t+1$ ", or equivalently $\mathcal{E}_{k}:=\left\{x_{u}^{(t+1)} \neq \sigma\right\}$, where $k$ is that of $(k, p, \sigma)$-MAJORITY. It holds that $\mathbf{P}\left(\mathcal{E}_{2 h+1} \mid \mathbf{x}^{(t)}=\overline{\mathbf{x}}\right)=\mathbf{P}\left(\mathcal{E}_{2 h+2} \mid \mathbf{x}^{(t)}=\overline{\mathbf{x}}\right)$, for every $h \in \mathbb{N}_{0}$. 
Remark 1. Consider a graph with any initial configuration $\mathbf{x}^{(0)}$. Fix any $p \in[0,1]$ and $\sigma \in$ $\{\mathcal{R}, \mathcal{B}\}$. Proposition 5.11 implies that, for every $h \in \mathbb{N}_{0}$, the $(2 h+1, p, \sigma)$-MAJORITY and the $(2 h+2, p, \sigma)$-MAJORITY follow the same law and, thus, their evolution is the same.

\section{Limit Cases: Voter and Deterministic Majority Dynamics}

In this section we analyze the two limit cases of $(k, p, \sigma)$-MAJORITY, considering the case $k=1$ as well as the case in which $k$ is large. In particular, 1-MAJORITY is equivalent to VOTER, i.e., nodes copy the state of a randomly sampled neighbor. In Section 6.1 we analyze its behavior on the biased communication model described in Section 3 . On the other hand, for large values of $k$, one might expect a similar behavior to that of DETERMINISTIC MAJORITY, in which nodes update their state to that supported by the majority of nodes in their entire neighborhood. In Section 6.2 we investigate the relation between the two dynamics in our biased communication model.

\subsection{Voter Dynamics}

Differently from the general case of Theorem 5.1 where $k \geq 3$, no phase transition is observed for $(1, p, \mathcal{B})$-MAJORITY, due to the linearity of the dynamics. Moreover, the effect of the bias $p$ has a strong impact on its behavior. While the standard binary voter dynamics needs $\Omega(n)$ rounds to reach a consensus on one of the two states, e.g., on the complete graph, we show in Proposition 6.1 that in the biased communication model a $\mathcal{B}$-almost-consensus is reached in $\mathcal{O}(1)$ rounds, w.h.p., regardless of the initial configuration. In other words, in a constant number of rounds any potential majority is subverted to a majority on the state promoted by the bias. In the complete graph, for example, this would imply a convergence to a consensus in $\mathcal{O}(\log n)$ rounds $4^{4}$ i.e., exponentially faster than in the classic scenario with no bias.

Proposition 6.1. Consider a sequence of graphs $\left(G_{n}\right)_{n \in \mathbb{N}}$ such that $\min _{u \in V} \delta_{u}=\omega(\log n)$. For any fixed $n$, consider the $(1, p, \mathcal{B})$-MAJORITY dynamics with any initial configuration $\mathbf{x}^{(0)}$ and $p>0$ constant. The process reaches a $\mathcal{B}$-almost-consensus within $\mathcal{O}(1)$ rounds, w.h.p.

The proof is essentially the same as that one of the fast convergence regime in Theorem 5.1. It can be found in Section 10.1

\subsection{Deterministic Majority Dynamics}

As mentioned earlier, one might expect that as $k$ grows $(k, p, \sigma)$-MAJORITY would behave similarly to DETERMINISTIC MAJORITY in the biased communication model, which we denote with $(p, \sigma)$-Deterministic MAJORITy. We make this link rigorous in Propositions 6.2 and 6.3 . In particular, we show that, if the graph satisfies the density assumption $\min _{u} \delta_{u}=\omega(\log n)$, the $(p, \sigma)$-DETERMINISTIC MAJORITY has a sharp phase transition at $p=\frac{1}{2}$, that is the limit as $k \rightarrow \infty$ of the critical value $p_{k}^{\star}$ (as proved in Lemma 8.3).

For the sake of readability, we restrict the analysis of this section to the case in which the initial configuration in which every vertex is in state $\mathcal{R}$.

Proposition 6.2. Consider a sequence of graphs $\left(G_{n}\right)_{n \in \mathbb{N}}$ such that $\min _{u \in V} \delta_{u}=\omega(\log n)$. For any fixed $n$, consider the $(p, \mathcal{B})$-DETERMINISTIC MAJORITY dynamics. Then:

- if $p=\frac{1}{2}+c$ for some $c>0$, then

$$
\mathbf{P}\left(\operatorname{vol}\left(B^{(1)}\right)=\operatorname{vol}(V)\right)=1-o(1) ;
$$

\footnotetext{
${ }^{4}$ The specific graph topology allows to use our arguments until a complete consensus.
} 
- if $p=\frac{1}{2}-c$ for some $c>0$, then for all $K>0$

$$
\mathbf{P}\left(\forall t \leq n^{K}, \operatorname{vol}\left(R^{(t)}\right)=\operatorname{vol}(V)\right)=1-o(1) .
$$

The proof of Proposition 6.2 is postponed to Section 10.2 .

Note that the behavior of the $(p, \mathcal{B})$-DETERMINISTIC MAJORITY is much stronger than the usual almost sense we used throughout the paper. In fact, if $p>\frac{1}{2}$ by a constant quantity, the $\mathcal{B}$-consensus is reached in a single round with high probability. On the other hand, if $p<\frac{1}{2}$, the initial $\mathcal{R}$-consensus lasts for any polynomial number of rounds.

In Proposition 6.3 we show that the same behavior holds - in a weaker sense-for $(k, p, \mathcal{B})$ MAJORITY, for all sufficiently large values of $k$. For the sake of clarity, in the next proposition we will use the notation $\mathbf{P}_{k}$ to denote the law of $(k, p, \mathcal{B})$-MAJORITY for a given value of $k$.

Proposition 6.3. Consider a sequence of graphs $\left(G_{n}\right)_{n \in \mathbb{N}}$ such that $\min _{u \in V} \delta_{u}=\omega(\log n)$. For any fixed $n$, consider the $(k, p, \mathcal{B})$-MAJORITY dynamics. Let $\left|p-\frac{1}{2}\right|=c$ for some $c>0$. Then for all $\gamma>0$ there exists $H=H(c, \gamma)$ such that:

- if $p>\frac{1}{2}$ then

$$
\mathbf{P}_{k}\left(\frac{\operatorname{vol}\left(B^{(1)}\right)}{\operatorname{vol}(V)} \geq 1-\gamma\right)=1-o(1), \quad \forall k>H
$$

- if $p<\frac{1}{2}$ then

$$
\mathbf{P}_{k}\left(\forall t \in\left[0, n^{K}\right], \frac{\operatorname{vol}\left(R^{(t)}\right)}{\operatorname{vol}(V)} \geq 1-\gamma\right)=1-o(1), \quad \forall k>H, \forall K>0 .
$$

The proof is in Section 10.2. We point out that the same behavior of Proposition 6.2 can be achieved for $(k, p, \mathcal{B})$-MAJORITY without any topological assumption, by just letting $k$ grow with $n$ as $k=\omega(\log n)$.

\section{Discussion and Outlook}

The biased communication model described in Section 3 and analyzed throughout the paper for the $k$-MAJORITY dynamics allows us to reason about the strength of consensus against adversarial nodes with a simple framework already presented in [CNNS18. In particular, this is possible for a class of graphs known as volume-regular graphs, recently introduced in [BCPR19], that are strictly related to ordinary lumpable Markov Chains [KS60].

In the following we first introduce such a class of graphs, of which notable examples are graphs sampled from the regular Stochastic Block Model [HLL83], and then discuss potential applications of the biased communication model in more general scenarios.

Definition 7.1 (Volume-regular graphs [BCPR19]). Let $G=(V, E)$ be a graph, and let $\mathcal{V}=$ $\left\{V_{1}, \ldots, V_{\ell}\right\}$ be an $\ell$-partition of the vertex set $V$. Let us call $\delta(u)$ the degree of node $u$ and $\delta_{i}(u)$ the number of neighbors that node $u$ has toward $V_{i}$. The graph $G$ is volume-regular w.r.t. $\mathcal{V}$ if for every pair $i, j \in\{1, \ldots, \ell\}$ and for every pair of nodes $u, v \in V_{i}$, it holds that $\frac{\delta_{j}(u)}{\delta(u)}=\frac{\delta_{j}(v)}{\delta(v)}$, i.e., every pair of nodes in each set $V_{i}$ of the partition $\mathcal{V}$ has the same fraction of neighbors toward any other set $V_{j}$ of the partition $\mathcal{V}$. 
Suppose one of the sets, $V_{i}$, in the partition $\mathcal{V}$ of a volume-regular graph $G$ has reached a local consensus on state $\mathcal{R}$ while running the $k$-MAJORITY dynamics. Now consider the $k$-MAJORITY dynamics in the biased communication model described above, where with probability $p$ each sample is seen in state $\mathcal{B}$, and run on the subgraph induced by $V_{i}$. If one considers $p=\frac{\delta(u)-\delta_{i}(u)}{\delta(u)}$, for any node $u \in V_{i}$, then the process is completely equivalent to a scenario in which the nodes in $V_{i}$ are trying to maintain their local consensus while all the others are in the opposite state.

In this setting, our results in Theorems 5.1 and 5.7 shed light on the relation between the topology of the network, i.e., the cut between $V_{i}$ and the rest of the network, and the number of nodes in the sample, $k$, in order for the nodes in $V_{i}$ to "resist" against all the others that are pushing them toward changing state. In this sense, our results could be exploited to prove similar phenomena in other topologies, e.g., akin to what has been done in [CNNS18, CNS19, SS19] for 2-CHOICES and 3-MAJORITY.

As already noted earlier, a stronger assumption on the minimum degree, i.e., considering graphs with minimum degree $\Omega(n)$, would be sufficient to prove a full consensus on the state $\sigma$ promoted by the bias, in $\mathcal{O}(\log n)$ rounds, in all the scenarios in which we prove a $\sigma$-almostconsensus. Graphs satisfying such assumption are not necessarily graphs with strong expansion properties, e.g., consider two equal-sized cliques connected by a single edge. It remains unclear whether it is possible to prove similar results for sparser topologies and which are the minimum topological assumptions that are necessary to prove a full consensus. More precisely, it would be interesting to see whether how our results can be sharpened by assuming a particular topology as, e.g., an Erdős-Rényi random graph $G(n, p)$ with $p=\frac{\omega(\log n)}{n}$.

From the statistical physics perspective, it would also be of interest the analysis of the critical case $p=p_{k}^{\star}$ on some particular topologies. In particular, it would be notable if precise asymptotics on the convergence time could be obtained in the critical regime without any topological assumption on the underlying graph.

Finally, possible research directions that could lead to non-obvious conclusions are that of applying our biased framework to other dynamics or to consider more than two states.

\section{Proofs of Section 4}

Recall from Definition 4.1 that $F_{p, k}(x):=\mathbf{P}\left(\operatorname{Bin}(k,(1-p) x) \geq \frac{k+1}{2}\right)$ for every odd $k$. In this section we study the fixed points of function $F_{p, k}(x)$, i.e., we want to solve the equation $F_{p, k}(x)=x$ for every $p \in(0,1)$. We first consider the simplest case $k=3$.

Lemma 8.1. Consider the equation $F_{p, 3}(x)=x$. It holds that:

- if $p<\frac{1}{9}$ then there exist $\varphi^{-}, \varphi^{+} \in\left[\frac{1}{2(1-p)}, 1\right]$ such that $F_{p, 3}(x)=x$ has solution $0, \varphi^{-}$, and $\varphi^{+}$, with $\varphi^{-}<\varphi^{+}$;

- if $p=\frac{1}{9}$ then there exists $\varphi \in\left[\frac{1}{2(1-p)}, 1\right]$ such that $F_{p, 3}(x)=x$ has solution 0 and $\varphi$;

- if $p>\frac{1}{9}$ then $F_{p, 3}(x)=x$ has 0 as unique solution.

Proof. With $k=3$ we have that

$$
\begin{aligned}
F_{p, 3}(x) & =(1-p)^{3} x^{3}-3(1-p)^{2} x^{2}[1-(1-p) x] \\
& =-2(1-p)^{3} x^{3}+3(1-p)^{2} x^{2} .
\end{aligned}
$$

With some algebraic manipulations on the equation $F_{p, 3}(x)=x$, we get that

$$
F_{p, 3}(x)=x \quad \Longleftrightarrow \quad x\left[2(1-p)^{3} x^{2}-3(1-p)^{2} x+1\right]=0 .
$$


Note that Eq. (12) has solutions different from 0 if and only if $2(1-p)^{3} x^{2}-3(1-p)^{2} x+1=0$ has at least one solution, i.e., if the discriminant $\Delta=(1-p)^{3}(1-9 p) \geq 0$. If $p=\frac{1}{9}$ then $\Delta=0$ and thus the unique solution of Eq. (12) different from 0 is

$$
\varphi=\frac{27}{32}>\frac{9}{16}=\frac{1}{2(1-p)} \geq \frac{1}{2} .
$$

If $p<\frac{1}{9}$, instead, Eq. 12 has two solutions different from 0 which are

$$
\varphi^{ \pm}=\frac{3(1-p)^{2} \pm \sqrt{(1-p)^{3}(1-9 p)}}{4(1-p)^{3}} \geq \frac{1}{2(1-p)} \geq \frac{1}{2} .
$$

We now look at the function $F_{p, k}$ for generic values of $k$. Before stating and proving the version of Lemma 8.1 for generic $k$, we show a set of properties of the function $F_{p, k}$ in the following Note 1 and Claims 1 to 4

Note 1. Recall that $F_{p, k}(x):=\mathbf{P}\left(\operatorname{Bin}(k,(1-p) x) \geq \frac{k+1}{2}\right)$. Note that:

1. $F_{p, k}(0)=0$ for every $p \in(0,1)$ and every odd $k$.

2. $F_{p, k}(1)<1$ for every $p \in(0,1)$ and every odd $k$.

3. $F_{p, k}(x)$ is a continuous function in the variable $x$ for every $p \in(0,1)$ and every $k$.

4. $F_{p, k}(x)$ is increasing in the variable $x$ for every $p \in(0,1)$ and every odd $k$. In fact, if we take $x<y$, we have that

$$
\mathbf{P}\left(\operatorname{Bin}(k,(1-p) x) \geq \frac{k+1}{2}\right)<\mathbf{P}\left(\operatorname{Bin}(k,(1-p) y) \geq \frac{k+1}{2}\right) .
$$

5. $F_{p, k}(x)$ is continuous and decreasing in $p$ for any $x \in[0,1]$ and for every odd $k$.

6. If $k$ is odd and $p<\frac{1}{2}$, we have $\frac{1}{2(1-p)} \in[0,1]$ and $F_{p, k}\left(\frac{1}{2(1-p)}\right)=\frac{1}{2}$. In fact,

$$
\begin{aligned}
F_{p, k}\left(\frac{1}{2(1-p)}\right) & =\mathbf{P}\left(\operatorname{Bin}\left(k, \frac{1}{2}\right) \geq \frac{k+1}{2}\right)=\sum_{i=(k+1) / 2}^{k}\left(\begin{array}{c}
k \\
i
\end{array}\right)\left(\frac{1}{2}\right)^{i}\left(\frac{1}{2}\right)^{k-i} \\
& =\left(\frac{1}{2}\right)^{k} \sum_{i=(k+1) / 2}^{k}\left(\begin{array}{c}
k \\
i
\end{array}\right) \stackrel{(a)}{=}\left(\frac{1}{2}\right)^{k} \frac{2^{k}}{2}=\frac{1}{2}
\end{aligned}
$$

where in (a) we use that $\left(\begin{array}{c}k \\ i\end{array}\right)=\left(\begin{array}{c}k \\ k-i\end{array}\right)$ and $\sum_{i=0}^{k}\left(\begin{array}{c}k \\ i\end{array}\right)=2^{k}$.

7. $F_{p, k}\left(\frac{1}{2}\right) \leq \frac{1}{2}$ for every $p \in[0,1]$ and every odd $k$. In fact, by Item 4

$$
F_{p, k}\left(\frac{1}{2}\right) \leq F_{p, k}\left(\frac{1}{2(1-p)}\right)=\frac{1}{2}
$$

Claim 1. The function $F_{p, k}(x)$ is non-decreasing in $k$ for every $x \in\left[\min \left\{\frac{1}{2(1-p)}, 1\right\}, 1\right]$ and $p \in[0,1]$, while it is non-increasing in $k$ for every $x \in\left[0, \min \left\{\frac{1}{2(1-p)}, 1\right\}\right]$ and $p \in[0,1]$. 
Proof. Let $X$ and $Y$ be two random variables with laws $\operatorname{Bin}(2 h+1, q)$ and $\operatorname{Bin}(2 h+3, q)$, respectively. In the following we prove that

$$
\begin{aligned}
& \mathbf{P}(Y \geq h+2) \geq \mathbf{P}(X \geq h+1) \text { if } q \geq \frac{1}{2}, \\
& \mathbf{P}(Y \geq h+2) \leq \mathbf{P}(X \geq h+1) \text { if } q \leq \frac{1}{2} .
\end{aligned}
$$

Note that

$$
\begin{aligned}
\mathbf{P}(Y \geq h+2) & =\mathbf{P}(X \geq h+2)+\mathbf{P}(X=h+1)\left(1-(1-q)^{2}\right)+\mathbf{P}(X=h+1) q^{2} \\
& =\mathbf{P}(X \geq h+2)+2 q \mathbf{P}(X=h+1) .
\end{aligned}
$$

Now we compute $\mathbf{P}(Y \geq h+2)-\mathbf{P}(X \geq h+1)$ by using Eq. (15). We get that

$$
\begin{aligned}
& \mathbf{P}(Y \geq h+2)-\mathbf{P}(X \geq h+1)=\mathbf{P}(X \geq h+2)+2 q \mathbf{P}(X=h+1)-\mathbf{P}(X \geq h+1) \\
& =\mathbf{P}(X \geq h+2)+2 q \mathbf{P}(X=h+1)-\mathbf{P}(X=h+1)-\mathbf{P}(X \geq h+2) \\
& =(2 q-1) \mathbf{P}(X=h+1)
\end{aligned}
$$

whose sign depends only on $(2 q-1)$. We can conclude that $\mathbf{P}(Y \geq h+2) \geq \mathbf{P}(X \geq h+1)$ for every $q \geq \frac{1}{2}$ and $\mathbf{P}(Y \geq h+2) \leq \mathbf{P}(X \geq h+1)$ for every $q \leq \frac{1}{2}$.

Let $k=2 h+1$. From Eqs. (13) and (14) we have that $F_{p, k}(x)$ is increasing in $k$ for every $x \geq \frac{1}{2(1-p)}$ while it is decreasing in $k$ for every $x \leq \frac{1}{2(1-p)}$.

Claim 2. It holds that

$$
\frac{d}{d x} F_{p, k}(x)=k(1-p) \mathbf{P}\left(\operatorname{Bin}(k-1,(1-p) x)=\frac{k-1}{2}\right) .
$$

Proof. We start by showing that

$$
\frac{d}{d u} \mathbf{P}\left(\operatorname{Bin}(k, u) \leq \frac{k-1}{2}\right)=-k \mathbf{P}\left(\operatorname{Bin}(k-1, u)=\frac{k-1}{2}\right) .
$$

Observe that

$$
\begin{aligned}
\frac{d}{d u} \mathbf{P}\left(\operatorname{Bin}(k, u) \leq \frac{k-1}{2}\right) & =\sum_{i=0}^{\frac{k-1}{2}}\left(\begin{array}{c}
k \\
i
\end{array}\right) \frac{d}{d u}\left[u^{i}(1-u)^{k-i}\right] \\
& =\frac{d}{d u}\left[(1-u)^{k}\right]+\sum_{i=1}^{\frac{k-1}{2}}\left(\begin{array}{c}
k \\
i
\end{array}\right) \frac{d}{d u}\left[u^{i}(1-u)^{k-i}\right] .
\end{aligned}
$$

We consider separately the term $\frac{d}{d u}\left[u^{i}(1-u)^{k-i}\right]$ for $i \geq 1$. We have

$$
\frac{d}{d u}\left[u^{i}(1-u)^{k-i}\right]=i u^{i-1}(1-u)^{k-i}-(k-i) u^{i}(1-u)^{k-1-i} .
$$

Note that

$$
\begin{aligned}
\sum_{i=1}^{\frac{k-1}{2}}\left(\begin{array}{l}
k \\
i
\end{array}\right) i u^{i-1}(1-u)^{k-i} & =\sum_{i=1}^{\frac{k-1}{2}} \frac{k !}{(i-1) !(k-i) !} u^{i-1}(1-u)^{k-i} \\
& \stackrel{(j=i-1)}{=} \sum_{j=0}^{\frac{k-3}{2}} \frac{k !}{j !(k-1-j) !} u^{j}(1-u)^{k-1-j}
\end{aligned}
$$


and

$$
\sum_{i=1}^{\frac{k-1}{2}}\left(\begin{array}{l}
k \\
i
\end{array}\right)(k-i) u^{i}(1-u)^{k-1-i}=\sum_{i=1}^{\frac{k-1}{2}} \frac{k !}{i !(k-1-i) !} u^{i}(1-u)^{k-1-i} .
$$

So by Eqs. (16) to 19) we get

$$
\begin{aligned}
& \frac{d}{d u} \mathbf{P}\left(\operatorname{Bin}(k, u) \leq \frac{k-1}{2}\right)=\frac{d}{d u}\left[(1-u)^{k}\right]+\sum_{i=1}^{\frac{k-1}{2}}\left(\begin{array}{c}
k \\
i
\end{array}\right) \frac{d}{d u}\left[u^{i}(1-u)^{k-i}\right] \\
& =-k(1-u)^{k-1}+\sum_{j=0}^{\frac{k-3}{2}} \frac{k !}{j !(k-1-j) !} u^{j}(1-u)^{k-1-j}-\sum_{i=1}^{\frac{k-1}{2}} \frac{k !}{i !(k-1-i) !} u^{i}(1-u)^{k-1-i} \\
& =-k(1-u)^{k-1}+k(1-u)^{k-1}-\frac{k !}{\left(\frac{k-1}{2}\right) !\left(\frac{k-1}{2}\right) !} u^{\frac{k-1}{2}}(1-u)^{\frac{k-1}{2}} \\
& =-k\left(\begin{array}{c}
k-1 \\
\frac{k-1}{2}
\end{array}\right) u^{\frac{k-1}{2}}(1-u)^{\frac{k-1}{2}}=-k \mathbf{P}\left(\operatorname{Bin}(k-1, u)=\frac{k-1}{2}\right) .
\end{aligned}
$$

We want to use Eq. (20) for computing the derivative of $F_{p, k}(x)$. Note that

$$
\begin{aligned}
\frac{d}{d x} F_{p, k}(x) & =\frac{d}{d x}\left(1-\mathbf{P}\left(\operatorname{Bin}(k,(1-p) x)<\frac{k+1}{2}\right)\right) \\
& =-\frac{d}{d x}\left(\mathbf{P}\left(\operatorname{Bin}(k,(1-p) x) \leq \frac{k-1}{2}\right)\right) .
\end{aligned}
$$

If we call $u(x)=(1-p) x$ we have that $\frac{d}{d x} F_{p, k}(x)=\frac{d}{d u}\left(F_{p, k}(u)\right) \frac{d}{d x}(u(x))$. Thus, by Eq. 21) we can conclude that

$$
\begin{aligned}
\frac{d}{d x} F_{p, k}(x) & =\frac{d}{d u}\left(F_{p, k}(u)\right) \frac{d}{d x}(u(x))=-\frac{d}{d u}\left(\mathbf{P}\left(\operatorname{Bin}(k, u) \leq \frac{k-1}{2}\right)\right)(1-p) \\
& =k(1-p) \mathbf{P}\left(\operatorname{Bin}(k-1,(1-p) x)=\frac{k-1}{2}\right) .
\end{aligned}
$$

Claim 3. It holds that

$$
\frac{d^{2}}{d x^{2}} F_{p, k}(x)=k(k-1)(1-p)^{3}\left(\begin{array}{c}
k-2 \\
\frac{k-1}{2}
\end{array}\right)\left(x-(1-p) x^{2}\right)^{\frac{k-3}{2}}(1-2(1-p) x) .
$$

Proof. We start by showing that

$$
\frac{d}{d u}\left(\mathbf{P}\left(\operatorname{Bin}(k-1, u)=\frac{k-1}{2}\right)\right)=(k-1)\left(\begin{array}{c}
k-2 \\
\frac{k-1}{2}
\end{array}\right)\left(u-u^{2}\right)^{\frac{k-3}{2}}(1-2 u) .
$$

Note that

$$
\begin{aligned}
\frac{d}{d u}\left(\mathbf{P}\left(\operatorname{Bin}(k-1, u)=\frac{k-1}{2}\right)\right) & =\left(\begin{array}{c}
k-1 \\
\frac{k-1}{2}
\end{array}\right) \frac{d}{d u}\left[u^{\frac{k-1}{2}}(1-u)^{\frac{k-1}{2}}\right] \\
& =\left(\begin{array}{c}
k-1 \\
\frac{k-1}{2}
\end{array}\right) \frac{d}{d u}\left(u-u^{2}\right)^{\frac{k-1}{2}} \\
& =\left(\begin{array}{c}
k-1 \\
\frac{k-1}{2}
\end{array}\right) \frac{k-1}{2}\left(u-u^{2}\right)^{\frac{k-3}{2}}(1-2 u)
\end{aligned}
$$




$$
=(k-1)\left(\begin{array}{c}
k-2 \\
\frac{k-1}{2}
\end{array}\right)\left(u-u^{2}\right)^{\frac{k-3}{2}}(1-2 u) .
$$

If we call $u(x)=(1-p) x$ we can compute the second derivative of $F_{p, k}(x)$ by using Eq. 22. Indeed,

$$
\begin{aligned}
\frac{d^{2}}{d x^{2}} F_{p, k}(x) & =k(1-p) \frac{d}{d x}\left(\mathbf{P}\left(\operatorname{Bin}(k-1,(1-p) x)=\frac{k-1}{2}\right)\right) \\
& =k(1-p) \frac{d}{d u}\left(\mathbf{P}\left(\operatorname{Bin}(k-1, u)=\frac{k-1}{2}\right)\right) \frac{d}{d x}(u(x)) \\
& =k(k-1)(1-p)^{2}\left(\begin{array}{c}
k-2 \\
\frac{k-1}{2}
\end{array}\right)\left((1-p) x-(1-p)^{2} x^{2}\right)^{\frac{k-3}{2}}(1-2(1-p) x) \\
& =k(k-1)(1-p)^{3}\left(\begin{array}{c}
k-2 \\
\frac{k-1}{2}
\end{array}\right)\left(x-(1-p) x^{2}\right)^{\frac{k-3}{2}}(1-2(1-p) x) .
\end{aligned}
$$

Claim 4. For $p \in(0,1)$ and for any odd $k$, the map $x \mapsto F_{p, k}(x)$ is a convex function for $x \in\left[0, \min \left\{\frac{1}{2(1-p)}, 1\right\}\right)$.

Proof. By direct computation:

$$
[x(1-(1-p) x)]^{\frac{k-3}{2}}(1-2(1-p) x)>0 \Longleftrightarrow 1-2(1-p) x>0 \Longleftrightarrow x<\frac{1}{2(1-p)} .
$$

We are now ready to state and prove the generalized version of Lemma 8.1.

Lemma 4.2. For every finite odd $k \geq 3$, there exists $p_{k}^{\star} \in\left[\frac{1}{9}, \frac{1}{2}\right)$ such that:

- if $p<p_{k}^{\star}$, there exist $\varphi_{p, k}^{-}, \varphi_{p, k}^{+} \in\left(\frac{1}{2(1-p)}, 1\right]$ with $\varphi_{p, k}^{-}<\varphi_{p, k}^{+}$such that $F_{p, k}(x)=x$ has solutions $0, \varphi_{p, k}^{-}$, and $\varphi_{p, k}^{+}$;

- if $p=p_{k}^{\star}$, there exists $\varphi_{p, k} \in\left(\frac{1}{2(1-p)}, 1\right]$ such that $F_{p, k}(x)=x$ has solutions 0 and $\varphi_{p, k}$;

- if $p>p_{k}^{\star}$, then $F_{p, k}(x)=x$ has 0 as unique solution.

Moreover the sequence $\left\{p_{k}^{\star}\right\}_{k}$ is increasing.

Proof. Recall that, by Claim 4, the map $x \mapsto F_{p, k}(x)$ is convex in $(0,1)$ for all $p \in(1 / 2,1)$ and $k$ odd. Moreover, being $F_{p, k}(0)=0$ (Item 1 in Note 1) and $F_{p, k}(1)<1$ (Item 2 in Note 1), regardless of the value of $k$ there are no solutions to the equation in the variable $x$

$$
F_{p, k}(x)=x,
$$

as soon as $p>1 / 2$. By Item 6 in Note 1 we know that, for all $k$ odd and $p \in(0,1 / 2)$,

$$
F_{p, k}\left(\frac{1}{2(1-p)}\right)=\frac{1}{2}<\frac{1}{2(1-p)} .
$$

Therefore, for all $k$ odd and $p \in(0,1)$ there are no solutions in $\left(0, \frac{1}{2(1-p)}\right]$ to the equation

$$
F_{p, k}(x)=x
$$

since it holds $F_{p, k}(x)<x$.

Hence, we now look for possible solutions in the interval $\left(\frac{1}{2(1-p)}, 1\right)$. The case $k=3$ has been studied in Lemma 8.1. Note that 

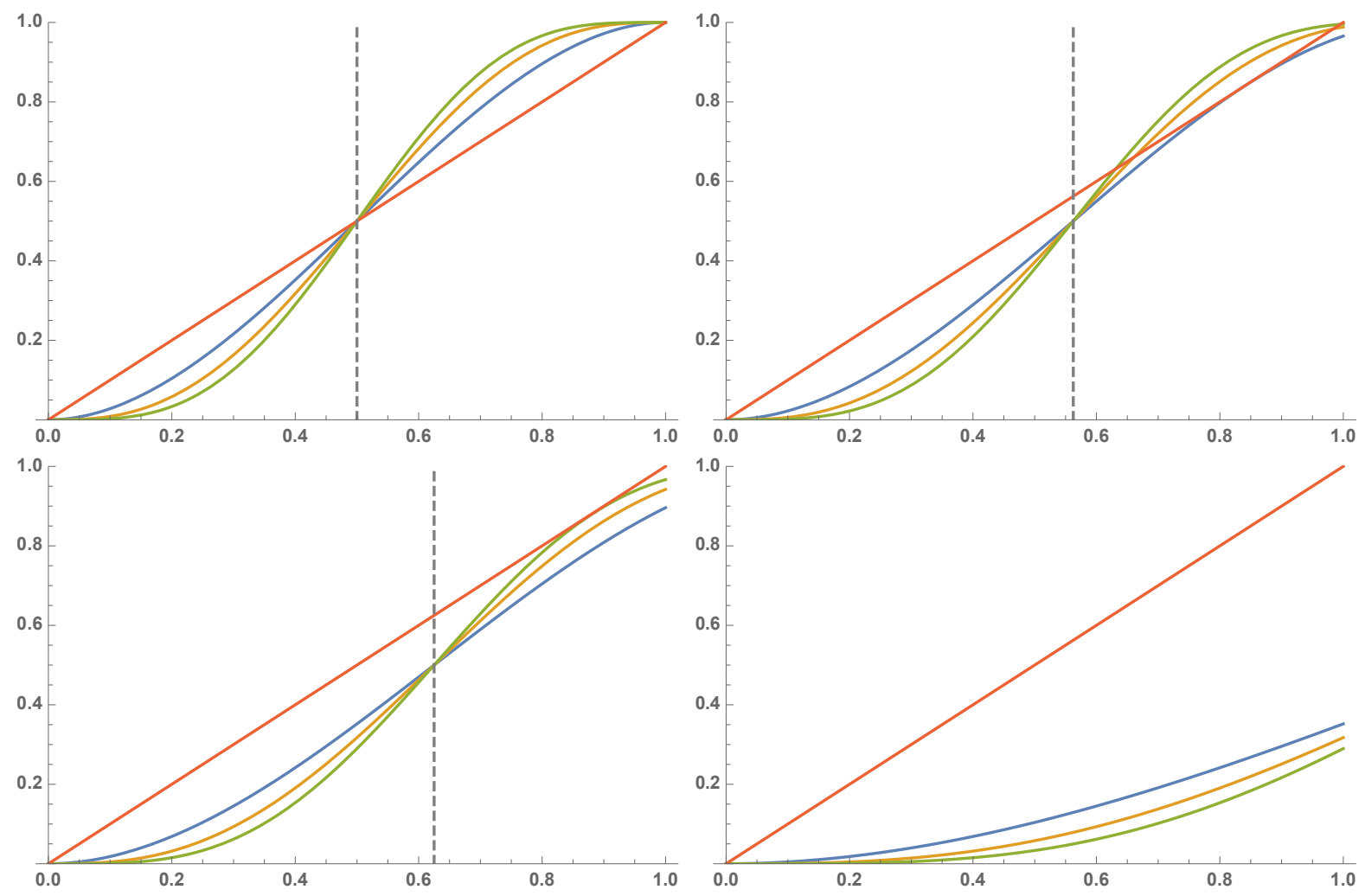

Figure 2: Plot of the function $x \mapsto F_{p, k}(x)$. The blue, orange, and green curves represent the cases $k=3,5$ and 7 , respectively. From the top left corner in clockwise order we have the cases $p=0, \frac{1}{9}, \frac{3}{5}$ and $\frac{1}{5}$, respectively. The dashed line has equation $x=1 /(2(1-p))$ and intersects the curves in their inflection point. The red line is the diagonal of the positive orthant.

(i) By Claim 1, the map $k \mapsto F_{p, k}(x)$ is increasing for all $p \in(0,1 / 2)$ and $x \in\left(\frac{1}{2(1-p)}, 1\right)$.

(ii) By Item 3 in Note 1 and by Claim 4 , the map $x \mapsto F_{p, k}(x)$ is continuous and concave in $\left(\frac{1}{2(1-p)}, 1\right)$ for all $k$ odd and $p \in(0,1 / 2)$.

(iii) By Item 5 in Note 1 , the map $p \mapsto F_{p, k}(x)$ is continuous and decreasing for all $k$ odd and $x \in(0,1)$.

Hence, by Item[i, for all $k>3$ there are two solutions in $(1 / 2,1)$ to the equation $F_{\frac{1}{9}, k}(x)=x$. Moreover, by Item ii and Item iii, for all $k>3$ there exists a value $p_{k}^{\star} \in\left(\frac{1}{9}, \frac{1}{2}\right)$ for which the solution to the equation $F_{p_{k}^{\star}, k}(x)=x$ is unique in the interval $\left(\frac{1}{2}, 1\right)$, while for $p>p_{k}^{\star}$ the equation $F_{p, k}(x)=x$, has no solutions in $(1 / 2,1)$.

Note that $F_{p, k}(1)<1$ (see Item 2 in Note 1) and at the beginning of the proof we have shown that $F_{p, k}(x)=x$ has no solutions in $\left(0, \frac{1}{2(1-p)}\right]$ for all $k$ odd and $p \in(0,1)$. Then we have that the possible solutions different from 0 of $F_{p, k}(x)=x$ are contained in the interval $\left(\frac{1}{2(1-p)}, 1\right) \subset(1 / 2,1)$.

Finally, by Item ii, we have that the sequence $\left(p_{k}^{\star}\right)_{k \in 2 \mathbb{N}+1}$ is increasing. 
Lemma 8.2. Assume $p=p_{k}^{\star}-c$ for some constant $c>0$. There exists a constant $\mu_{p, k} \in$ $\left(\varphi_{p, k}^{-}, \varphi_{p, k}^{+}\right)$such that, for all $x \in\left(\mu_{p, k}, 1\right]$, it holds that

$$
F_{p, k}^{\prime}(x) \in[0,1)
$$

Proof. By contradiction suppose that $F_{p, k}^{\prime}\left(\varphi_{p, k}^{+}\right) \geq 1$. Since $F_{p, k}^{\prime \prime}(x)<0$ for all $x \in\left(\frac{1}{2(1-p)}, 1\right)$, we have

$$
F_{p, k}^{\prime}(x)>F_{p, k}^{\prime}\left(\varphi_{p, k}^{+}\right) \geq 1, \quad \forall x \in\left(\frac{1}{2(1-p)}, \varphi_{p, k}^{+}\right) .
$$

Define $\psi(x):=F_{p, k}(x)-x$. By Eq. (23),

$$
\psi^{\prime}(x)=F_{p, k}^{\prime}(x)-1>0, \quad \forall x \in\left(\frac{1}{2(1-p)}, \varphi_{p, k}^{+}\right) .
$$

Hence,

$$
0=\psi\left(\varphi_{p, k}^{+}\right)>\psi(x), \quad \forall x \in\left(\frac{1}{2(1-p)}, \varphi_{p, k}^{+}\right) .
$$

In particular $\psi(x)<0$ implies

$$
F_{p, k}(x)<x, \quad \forall x \in\left(\frac{1}{2(1-p)}, \varphi_{p, k}^{+}\right) .
$$

On the other hand, the value $\varphi_{p, k}^{-}$lies in the interval $\left(\frac{1}{2(1-p)}, \varphi_{p, k}^{+}\right)$, and $F_{p, k}\left(\varphi_{p, k}^{-}\right)=\varphi_{p, k}^{-}$, which is in contradiction with Eq. (24). The claim follows by continuity of $F_{p, k}$.

In the following lemma we consider the limit of the critical bias $p_{k}^{\star}$ as $k \rightarrow \infty$.

Lemma 8.3. Consider the sequence $\left\{p_{k}^{\star}\right\}_{k \in 2 \mathbb{N}+1}$. Then we have

$$
\lim _{k \rightarrow \infty} p_{k}^{\star}=\frac{1}{2}
$$

Proof. Recall that

$$
F_{p, k}(x):=\mathbf{P}\left(\operatorname{Bin}(k,(1-p) x) \geq \frac{k+1}{2}\right) .
$$

We want to compute the following $\operatorname{limit}_{k \rightarrow \infty} \lim _{p, k}(x)$ for any $x \in(0,1) \backslash\left\{\frac{1}{2(1-p)}\right\}$, since $\lim _{k \rightarrow \infty} F_{p, k}\left(\frac{1}{2(1-p)}\right)=\frac{1}{2}$. Let us define $m_{p, x}$ and $s_{p, x}$, respectively the normalized expectation and variance of the random variable $\operatorname{Bin}(k,(1-p) x)$, that is

$$
m_{p, x}:=(1-p) x, \quad s_{p, x}:=(1-p) x(1-(1-p) x) .
$$

Note that

$$
\mathbf{P}\left(\operatorname{Bin}(k,(1-p) x) \geq \frac{k+1}{2}\right)=\mathbf{P}\left(\frac{\operatorname{Bin}(k,(1-p) x)-k m_{p, x}}{\sqrt{k s_{p, x}}} \geq \frac{k+1-2 k m_{p, x}}{2 \sqrt{k s_{p, x}}}\right)
$$

and by the Central Limit Theorem for $k \rightarrow+\infty$ the r.h.s. of Eq. 25 behaves like

$$
1-\Phi\left(\frac{k+1-2 k m_{p, x}}{2 \sqrt{k s_{p, x}}}\right)
$$

where $\Phi(x):=\mathbf{P}(Z \leq x)$ and $Z$ is a random variable with standard normal distribution. Since

$$
\lim _{k \rightarrow+\infty} \frac{k+1-2 k m_{p, x}}{2 \sqrt{k s_{p, x}}}=\lim _{k \rightarrow+\infty} \frac{\sqrt{k}(1-2(1-p) x)}{2 \sqrt{s_{p, x}}} \rightarrow \begin{cases}+\infty, & \text { if } x \in\left(0, \frac{1}{2(1-p)}\right), \\ -\infty, & \text { if } x \in\left(\frac{1}{2(1-p)}, 1\right)\end{cases}
$$


by Eqs. 25 to 27 we get that for any $x \in(0,1) \backslash\left\{\frac{1}{2(1-p)}\right\}$

$$
\lim _{k \rightarrow+\infty} F_{p, k}(x)= \begin{cases}0, & \text { if } x \in\left(0, \frac{1}{2(1-p)}\right), \\ 1, & \text { if } x \in\left(\frac{1}{2(1-p)}, 1\right) .\end{cases}
$$

In particular for any $\eta \in(0,1)$ and $x \in(0,1) \backslash\left\{\frac{1}{1+\eta}\right\}$ we have

$$
\lim _{k \rightarrow+\infty} F_{\frac{1-\eta}{2}, k}(x)= \begin{cases}0, & \text { if } x \in\left(0, \frac{1}{1+\eta}\right), \\ 1, & \text { if } x \in\left(\frac{1}{1+\eta}, 1\right) .\end{cases}
$$

Fix now $\eta \in(0,1)$. By Eq. $[29)$, for any $\gamma \in(0,1)$ there exists a constant $\bar{K}(\gamma)>0$ such that

$$
F_{\frac{1-\eta}{2}, k}\left(\frac{1}{1+\frac{\eta}{2}}\right)>1-\gamma, \quad \forall k>\bar{K}(\gamma)
$$

Fix $\gamma=\frac{\frac{\eta}{2}}{1+\frac{\eta}{2}}$. Hence by Eq. 30 there exists a constant $\bar{K}(\eta)>0$ such that

$$
F_{\frac{1-\eta}{2}, k}\left(\frac{1}{1+\frac{\eta}{2}}\right)>\frac{1}{1+\frac{\eta}{2}}, \quad \forall k>\bar{K}(\eta)
$$

and this implies

$$
p_{k}^{\star}>\frac{1-\eta}{2}, \quad \forall k>\bar{K}(\eta)
$$

So we have obtained that for any $\eta \in(0,1)$ there exists a constant $\bar{K}(\eta)>0$ such that $p_{k}^{\star}>\frac{1-\eta}{2}$ for all $k>\bar{K}(\eta)$. Moreover we know that $\left\{p_{k}^{\star}\right\}_{k \in \mathbb{N}}$ is an increasing sequence bounded from above by $\frac{1}{2}$ (see Lemma 4.2 . By these two facts we get the thesis.

\section{Proofs of Section 5}

Here we state and prove a generalized version of Proposition 5.2 .

Proposition 9.1. Consider $p=p_{k}^{\star}-c$, for some $c>0$. Hence the quantity $\varphi_{k, p}^{-}$in Lemma 4.2 is well defined. Consider $(k, p, \mathcal{B})$-MAJORITY starting from the initial configuration in which each vertex is $\mathcal{R}$ independently of the others, with probability $q \in\left[\mu_{p, k}+\eta, 1\right]$, for some sufficiently small $\eta>0$, where $\mu_{p, k}$ is defined as in Lemma 8.2. Then, for all $\gamma>0$,

$$
\mathbf{P}\left(\forall t \leq n^{K}, \forall v \in V, \quad \phi_{v}^{(t)} \in\left[q_{t}-\gamma, q_{t}+\gamma\right]\right)=1-o(1), \quad \forall K>0 .
$$

where the sequence $\left\{q_{t}\right\}_{t \geq 0}$ is defined recursively by

$$
q_{0}=q, \quad q_{t}=F_{k, p}\left(q_{t-1}\right) .
$$

Proof. For all $t \geq 0$ and $v \in V$ consider the events

$$
\mathcal{E}_{v}^{(t)}=\left\{\phi_{v}^{(t)} \in\left[q_{t}-\gamma, q_{t}+\gamma\right]\right\}, \quad \mathcal{E}^{(t)}=\bigcap_{v \in V} \mathcal{E}_{v}^{(t)}
$$

We start at $t=0$ by computing the probability that the event $\mathcal{E}^{(0)}$ occurs, i.e.,

$$
\mathbf{P}\left(\mathcal{E}^{(0)}\right)=\mathbf{P}\left(\forall v \in V, \phi_{u}^{(0)} \in[q-\gamma, q+\gamma]\right) .
$$


Fixed any $v \in V$, we look for a lower bound for the probability $\mathcal{E}_{v}^{(0)}$ which holds uniformly in $v \in V$.

$$
\begin{aligned}
\mathbf{P}\left(\mathcal{E}_{v}^{(0)}\right) & =\mathbf{P}\left(\frac{\sum_{w \in N_{v}} \mathbf{1}_{w \in R^{(0)}}}{\delta_{v}} \in[q-\gamma, q+\gamma]\right) \\
& =\mathbf{P}\left(\left|\sum_{w \in N_{v}} \mathbf{1}_{w \in R^{(0)}}-q \delta_{v}\right| \leq \gamma \delta_{v}\right) \\
& =\mathbf{P}\left(\left|\operatorname{Bin}\left(\delta_{v}, q\right)-q \delta_{v}\right| \leq \gamma \delta_{v}\right) \\
& =1-e^{-\omega(\log n)}
\end{aligned}
$$

where in the third equality we used the fact that

$$
\mathbf{P}\left(w \in R^{(0)}\right)=q, \quad \forall w \in V
$$

and that the events $\left\{w \in R^{(0)}\right\}$ and $\left\{u \in R^{(0)}\right\}$ are independent for $u \neq w$. Moreover, in the last asymptotic equality we used the classical multiplicative version of Chernoff's bound. By the density assumption $\min _{v \in V} \delta_{v}=\omega(\log (n))$, the lower bound above holds uniformly in $v \in V$, therefore, by the union bound

$$
\mathbf{P}\left(\mathcal{E}^{(0)}\right)=1-n \cdot e^{-\omega(\log n)}
$$

Now we take $t=1$, and we fix any configuration $\mathbf{x}^{(0)} \in \mathcal{E}^{(0)}$. Fixed any $v \in V$ we look for a lower bound for the conditional probability $\mathbf{P}\left(\mathcal{E}_{v}^{(1)} \mid \mathbf{x}^{(0)}\right)$, which holds uniformly in $v \in V$ and in $\mathbf{x}^{(0)} \in \mathcal{E}^{(0)}$. We start by rewriting

$$
\mathbf{P}\left(\mathcal{E}_{v}^{(1)} \mid \mathbf{x}^{(0)}\right)=\mathbf{P}\left(\left|\sum_{w \in N_{v}} \mathbf{1}_{w \in R^{(1)}}-q_{1} \delta_{v}\right| \leq \gamma \delta_{v} \mid \mathbf{x}^{(0)}\right) .
$$

Note that under any $\mathbf{x}^{(0)} \in \mathcal{E}^{(0)}$ the following stochastic domination holds

$$
\operatorname{Bin}\left(\delta_{v}, F_{p, k}(q-\gamma)\right) \preceq \sum_{w \in N_{v}} \mathbf{1}_{w \in R^{(1)}} \mid \mathbf{x}^{(0)} \preceq \operatorname{Bin}\left(\delta_{v}, F_{p, k}(q+\gamma)\right) .
$$

Hence, called

$$
X \sim \operatorname{Bin}\left(\delta_{v}, F_{p, k}(q-\gamma)\right), \quad Y \sim \operatorname{Bin}\left(\delta_{v}, F_{p, k}(q+\gamma)\right),
$$

it is sufficient to show that, for $Z=X, Y$, it holds that

$$
\mathbf{P}\left(\left|Z-q_{1} \delta_{v}\right| \leq \gamma \delta_{v}\right)=1-e^{-\omega(\log n)} .
$$

We show Eq. (33) for the case $Z=X$, being the proof for the case $Z=Y$ identical. By the triangle inequality and the fact that $q_{1}=F_{p, k}(q)$ we have

$$
\mathbf{P}\left(\left|X-q_{1} \delta_{v}\right| \leq \gamma \delta_{v}\right) \geq \mathbf{P}\left(\left|X-F_{p, k}(q-\gamma) \delta_{v}\right|+\left|F_{p, k}(q-\gamma)-F_{p, k}(q)\right| \delta_{v} \leq \gamma \delta_{v}\right) .
$$

By Lemma 8.2, for all $\eta>0$ and $x, y \in\left[\mu_{p, k}+\eta, 1\right]$ there exists some $L=L(\eta)<1$ such that

$$
\left|F_{p, k}(x)-F_{p, k}(y)\right| \leq L|x-y|,
$$

and in particular

$$
\left|F_{p, k}(q)-F_{p, k}(q-\gamma)\right| \leq L \gamma
$$


Hence, by Eqs. (34) and (35), we infer

$$
\mathbf{P}\left(\left|X-q_{1} \delta_{v}\right| \leq \gamma \delta_{v}\right) \geq \mathbf{P}\left(\left|X-F_{p, k}(q-\gamma)\right| \leq(1-L) \gamma \delta_{v}\right)=1-e^{-\omega(\log (n))} .
$$

Repeating the same argument with $Y$ instead of $X$, we deduce that Eq. (33) holds. Hence, by the uniformity of the argument in $v \in V$ and in $\mathbf{x}^{(0)} \in \mathcal{E}^{(0)}$, and by a union bound over $v \in V$ we obtain

$$
\mathbf{P}\left(\mathcal{E}^{(1)} \mid \mathcal{E}^{(0)}\right)=1-n \cdot e^{-\omega(\log (n))}
$$

Using the same techniques, this identity can be generalized for any $t \geq 0$, that is, for all $K>0$

$$
\begin{aligned}
\mathbf{P}\left(\forall v \in V, \forall t \in\left[0, n^{K}\right], \phi_{v}^{(t)} \in\left[q_{t}-\gamma, q_{t}+\gamma\right]\right) & =\mathbf{P}\left(\mathcal{E}^{(0)}\right) \prod_{s=1}^{n^{K}} \mathbf{P}\left(\mathcal{E}^{(s)} \mid \mathcal{E}^{(s-1)}\right) \\
& \geq 1-n^{K+1} \cdot e^{-\omega(\log (n))} .
\end{aligned}
$$

Notice that, if $q_{0}>\varphi_{p, k}^{+}, q_{t}$ converges to $\varphi_{p, k}^{+}$from above. On the other hand, if $q_{0} \in\left(\mu_{p, k}, \varphi_{p, k}^{+}\right)$ then for all $\gamma>0$ there exists some $T=T(\gamma)>0$ such that

$$
q_{t}>\varphi_{p, k}^{+}-\gamma, \quad \forall t \geq T
$$

and therefore $q_{t}$ converges to $\varphi_{p, k}^{+}$from below.

\subsection{Proof of Lemma 5.4}

Proof. Recall the formulation, given in Eq. (4), of the expected fraction of neighbors of a node $u$ that are in state $\mathcal{R}$ at round $t+1$. Being $p=p_{k}^{\star}+c$, it holds $F_{p, k}(x)<x$ for all $x \in[0,1]$, see Lemma 4.2. Moreover, the following claim holds true.

Claim 5. There exists a point $z_{p, k} \in[0,1]$ such that the linear function $r:[0,1] \rightarrow \mathbb{R}$, defined as

$$
r(x)=\frac{F\left(z_{p, k}\right)}{z_{p, k}} \cdot x
$$

is such that $r(x) \geq F_{p, k}(x)$. Moreover, the equality is attained only in two points, 0 and $z_{p, k} \in\left[\min \left\{\frac{1}{2(1-p)}, 1\right\}, 1\right]$.

Proof of Claim 5. Suppose that $p \geq \frac{1}{2}$. Then $F_{p, k}$ is a convex function in $[0,1]$. Hence

$$
F_{p, k}(x) \leq\left(F_{p, k}(1)-F_{p, k}(0)\right) x+F_{p, k}(0)=F_{p, k}(1) \cdot x .
$$

So, defining $z_{p, k}:=1$, we get the thesis in the case $p \geq \frac{1}{2}$.

Suppose now that $p<\frac{1}{2}$. Imposing the equality

$$
F_{p, k}(x)=r(x) \quad \Longleftrightarrow \quad \mathbf{P}\left(\operatorname{Bin}(k,(1-p) x) \geq \frac{k+1}{2}\right)=\frac{1-p}{1-p_{k}^{\star}} x,
$$

it is immediate that a solution is given by $x=0$. On the other hand, called $y=\frac{1-p}{1-p_{k}^{\star}} \cdot x$, the equation reads

$$
F_{p, k}\left(\frac{1-p_{k}^{\star}}{1-p} \cdot y\right)=y
$$

The latter can be rewritten as

$$
\mathbf{P}\left(\operatorname{Bin}\left(k,\left(1-p_{k}^{\star}\right) y\right) \geq \frac{k+1}{2}\right)=y \quad \Longleftrightarrow \quad F_{p_{k}^{\star}, k}(y)=y,
$$


which we know from Lemma 4.2 to have only a non-trivial solutions. Called $\bar{y} \neq 0$ such a solution, we have that

$$
z_{p, k}:=\frac{1-p_{k}^{\star}}{1-p} \cdot \bar{y}
$$

Note that $\bar{y}>\frac{1}{2(1-p)}$ and hence, since $\frac{1-p_{k}^{*}}{1-p}>1$, we have that $z_{p, k}>\frac{1}{2(1-p)}$.

By Claim 5 we have that

$$
F_{p, k}\left(\phi_{v}^{(t)}\right)<\frac{F_{p, k}\left(z_{p, k}\right)}{z_{p, k}} \phi_{v}^{(t)} \leq(1-\varepsilon) \phi_{v}^{(t)}
$$

where

$$
\varepsilon=\varepsilon(p, k):=\frac{1}{2}\left(1-\frac{F_{p, k}\left(z_{p, k}\right)}{z_{p, k}}\right) .
$$

Therefore, we can conclude the proof by just combining Eqs. (4) and (38), getting

$$
\mathbf{E}\left[\phi_{u}^{(t+1)} \mid \mathbf{x}^{(t)}=\overline{\mathbf{x}}\right]=\frac{1}{\delta_{u}} \sum_{v \in N_{u}} F_{p, k}\left(\phi_{v}^{(t)}\right) \leq \frac{1}{\delta_{u}} \sum_{v \in N_{u}}(1-\varepsilon) \phi_{v}^{(t)} \leq(1-\varepsilon) \phi_{\max }^{(t)} .
$$

\subsection{Proofs of Lemma 5.9 and Proposition 5.8}

Proof of Lemma 5.9. Let $x \in\left[0, \varphi_{p, k}^{-}-\eta\right]$ and consider the line

$$
r(x):=\frac{F_{p, k}\left(\varphi_{p, k}^{-}-\eta\right)}{\varphi_{p, k}^{-}-\eta} x .
$$

We have that $F_{p, k}(x)<r(x)$ for every $x \in\left(0, \varphi_{p, k}^{-}-\eta\right]$. Hence, by defining

$$
\varepsilon^{\prime}=\varepsilon^{\prime}(p, k, \eta):=\frac{1}{2}\left(1-\frac{F_{p, k}\left(\varphi_{p, k}^{-}-\eta\right)}{\varphi_{p, k}^{-}-\eta}\right),
$$

we get $F_{p, k}(x)<\left(1-\varepsilon^{\prime}\right) x$ for every $x \in\left(0, \varphi_{p, k}^{-}-\eta\right]$. We complete the proof of the claim by computing the conditional expectation, namely

$$
\mathbf{E}\left[\phi_{u}^{(t+1)} \mid \mathbf{x}^{(t)}=\overline{\mathbf{x}}\right]=\frac{1}{\delta_{u}} \sum_{v \in N_{u}} F_{p, k}\left(\phi_{v}^{(t)}\right)<\frac{1}{\delta_{u}} \sum_{v \in N_{u}}\left(1-\varepsilon^{\prime}\right) \phi_{v}^{(t)} \leq\left(1-\varepsilon^{\prime}\right) \phi_{\max }^{(t)} .
$$

Proof of Proposition 5.8. We start the proof by mimicking the proof of Proposition 9.1. Consider the sequence

$$
\rho_{t}:=(2 L)^{t} \rho_{0}
$$

where

$$
L:=\max _{x \in\left[\varphi_{p, k}^{-}, 1\right]} F_{p, k}^{\prime}(x) \geq 1
$$

and $\rho=\rho_{0}(\eta)$ will be defined later.

For all $t \geq 0$ and $v \in V$ consider the events

$$
\mathcal{E}_{v}^{(t)}=\left\{\phi_{v}^{(t)} \in\left[q_{t}-\rho_{t}, q_{t}+\rho_{t}\right]\right\}, \quad \mathcal{E}^{(t)}=\bigcap_{v \in V} \mathcal{E}_{v}^{(t)} .
$$


We start at $t=0$ by computing the probability that the event $\mathcal{E}^{(0)}$ occurs, i.e.,

$$
\mathbf{P}\left(\mathcal{E}^{(0)}\right)=\mathbf{P}\left(\forall v \in V, \phi_{u}^{(0)} \in\left[q-\rho_{0}, q+\rho_{0}\right]\right)=1-e^{\omega(\log n)} .
$$

As in the proof of Proposition 9.1, we start by taking $t=1$ and we fix any configuration $\mathbf{x}^{(0)} \in$ $\mathcal{E}^{(0)}$. Fixed any $v \in V$ we look for a lower bound for the conditional probability $\mathbf{P}\left(\mathcal{E}_{v}^{(1)} \mid \mathbf{x}^{(0)}\right)$, which holds uniformly in $v \in V$ and in $\mathbf{x}^{(0)} \in \mathcal{E}^{(0)}$. We start by rewriting

$$
\mathbf{P}\left(\mathcal{E}_{v}^{(1)} \mid \mathbf{x}^{(0)}\right)=\mathbf{P}\left(\left|\sum_{w \in N_{v}} \mathbf{1}_{w \in R^{(1)}}-q_{1} \delta_{v}\right| \leq \rho_{1} \delta_{v} \mid \mathbf{x}^{(0)}\right) .
$$

Note that under any $\mathbf{x}^{(0)} \in \mathcal{E}^{(0)}$ the following stochastic domination holds

$$
\operatorname{Bin}\left(\delta_{v}, F_{p, k}\left(q-\rho_{0}\right)\right) \preceq \sum_{w \in N_{v}} \mathbf{1}_{w \in R^{(1)}} \mid \mathbf{x}^{(0)} \preceq \operatorname{Bin}\left(\delta_{v}, F_{p, k}\left(q+\rho_{0}\right)\right) .
$$

Hence, called

$$
X \sim \operatorname{Bin}\left(\delta_{v}, F_{p, k}\left(q-\rho_{0}\right)\right), \quad Y \sim \operatorname{Bin}\left(\delta_{v}, F_{p, k}\left(q+\rho_{0}\right)\right),
$$

it is sufficient to show that, for $Z=X, Y$, it holds that

$$
\mathbf{P}\left(\left|Z-q_{1} \delta_{v}\right| \leq \rho_{1} \delta_{v}\right)=1-e^{-\omega(\log n)} .
$$

We show Eq. (41) for the case $Z=X$, being the proof for the case $Z=Y$ identical. By the triangle inequality and the fact that $q_{1}=F_{p, k}(q)$ we have

$$
\begin{aligned}
\mathbf{P}\left(\left|X-q_{1} \delta_{v}\right| \leq \rho_{1} \delta_{v}\right) & \geq \mathbf{P}\left(\left|X-F_{p, k}\left(q-\rho_{0}\right) \delta_{v}\right|+\left|F_{p, k}\left(q-\rho_{0}\right)-F_{p, k}(q)\right| \delta_{v} \leq \rho_{1} \delta_{v}\right) \\
& \geq \mathbf{P}\left(\left|X-F_{p, k}\left(q-\rho_{0}\right) \delta_{v}\right| \leq\left(\rho_{1}-L \rho_{0}\right) \delta_{v}\right) \\
& =\mathbf{P}\left(\left|X-F_{p, k}\left(q-\rho_{0}\right) \delta_{v}\right| \leq L \rho_{0} \delta_{v}\right) \\
& \geq 1-e^{-\omega(\log n)}
\end{aligned}
$$

and therefore by the uniformity of the argument in $v \in V$ and in $\mathbf{x}^{(0)} \in \mathcal{E}^{(0)}$, and by a union bound over $v \in V$

$$
\mathbf{P}\left(\mathcal{E}^{(1)} \mid \mathcal{E}^{(0)}\right)=1-n \cdot e^{-\omega(\log n)}
$$

Moreover, by repeating the same argument, we get that for any $t>0$

$$
\mathbf{P}\left(\left|\operatorname{Bin}\left(\delta_{v}, F_{p, k}\left(q_{t}-\rho_{t}\right)\right)-q_{t+1} \delta_{v}\right| \leq \rho_{t+1} \delta_{v}\right)=1-e^{-\omega(\log n)}
$$

and hence for all $t>0$ and $\mathbf{x}^{(t)} \in \mathcal{E}^{(t)}$

$$
\mathbf{P}\left(\mathcal{E}^{(t+1)} \mid \mathbf{x}^{(t)}\right)=1-e^{-\omega(\log n)} .
$$

Notice that, if $q_{0} \in\left(\varphi_{p, k}^{-}, \mu_{p, k}\right), q_{t}$ converges to $\varphi_{p, k}^{+}$from below and hence there exists some finite

$$
\tau=\tau_{p, k}:=\inf \left\{t \geq 0: q_{t}>\mu_{p, k}, \forall t>\tau\right\}<+\infty .
$$

Define the sequence

$$
g_{t}:=F_{p, k}\left(q_{t-1}-\rho_{t-1}\right) .
$$

We prove that $g_{t}$ is increasing and its increments are lower bounded by a constant uniformly in $t \leq \tau$. Let us start by noting that

$$
g_{t+1}-g_{t}=F_{p, k}\left(q_{t}-\rho_{t}\right)-F_{p, k}\left(q_{t-1}-\rho_{t-1}\right)
$$




$$
\begin{aligned}
& =F_{p, k}\left(q_{t}-\rho_{t}\right)-F_{p, k}\left(q_{t}\right)+F_{p, k}\left(q_{t}\right)-F_{p, k}\left(q_{t-1}\right)+F_{p, k}\left(q_{t-1}\right)-F_{p, k}\left(q_{t-1}-\rho_{t-1}\right) \\
& >F_{p, k}\left(q_{t}-\rho_{t}\right)-F_{p, k}\left(q_{t}\right)+F_{p, k}\left(q_{t}\right)-F_{p, k}\left(q_{t-1}\right) .
\end{aligned}
$$

Hence it is sufficient to show that

$$
F_{p, k}\left(q_{t}\right)-F_{p, k}\left(q_{t}-\rho_{t}\right)<F_{p, k}\left(q_{t}\right)-F_{p, k}\left(q_{t-1}\right) .
$$

Define

$$
C=\inf _{t \in[0, \tau]}\left(F_{p, k}\left(q_{t}\right)-F_{p, k}\left(q_{t-1}\right)\right)>0 .
$$

Then, we are left to show that the left hand side of Eq. (43) is strictly smaller than $C$. Note that

$$
F_{p, k}\left(q_{t}\right)-F_{p, k}\left(q_{t}-\rho_{t}\right) \leq L \rho_{t}=L \cdot(2 L)^{t} \rho_{0} \leq(2 L)^{\tau+1} \rho_{0} .
$$

In order for the latter to hold uniformly in $t \leq \tau$ it is sufficient to choose

$$
\rho_{0}:=\gamma \cdot \frac{C}{(2 L)^{\tau+1}}
$$

for any $\gamma \in(0,1)$. In conclusion, by Eq. 42, , for any $\gamma \in(0,1)$ we can deduce that

$$
\begin{aligned}
\mathbf{P}\left(\forall v \in V, \forall t \leq \tau, \phi_{v}^{(t)} \in\left[q_{t}-\gamma, q_{t}+\gamma\right]\right) & \geq \mathbf{P}\left(\mathcal{E}^{(0)}\right) \prod_{s=1}^{\tau} \mathbf{P}\left(\mathcal{E}^{(s)} \mid \mathcal{E}^{(s-1)}\right) \\
& \geq 1-\mathcal{O}\left(n^{-K}\right), \quad \forall K>0 .
\end{aligned}
$$

\subsection{Proof of Proposition 5.11}

Proof. Without loss of generality, let $\sigma=\mathcal{B}$ and, for simplicity, we omit the conditioning on $\mathbf{x}^{(t)}$. Hence, we rewrite the event $\mathcal{E}_{k}$ as "Node $u$ updates its state to $\mathcal{R}$ at round $t+1$ ". Let us focus on samples of odd size, i.e., $k=2 h+1$, where there cannot be ties. Let us define $z:=(1-p) \frac{\left|R_{u}^{(t)}\right|}{\delta_{u}}$, i.e., the probability that $u$ sees a given node in the sample in state $\mathcal{R}$, and the random variable $X_{R}=\operatorname{Bin}(2 h+1, z)$ modeling the number of nodes seen in state $\mathcal{R}$ out of a sample of size $2 h+1$. Since each sample is independent, one can look at the event $\mathcal{E}_{2 h+2}$ as performing an additional sample (with $z$ as success probability) after the first $2 h+1$ of the event $\mathcal{E}_{2 h+1}$. For this purpose, we also define the random variable $Y_{R}=\operatorname{Bin}(2 h+2, z)$. Therefore:

1. $\mathbf{P}\left(\mathcal{E}_{2 h+1}\right)=\mathbf{P}\left(X_{R} \geq h+1\right)=\mathbf{P}\left(X_{R}=h+1\right)+\mathbf{P}\left(X_{R}>h+1\right)$,

2. $\mathbf{P}\left(\mathcal{E}_{2 h+2}\right)=\mathbf{P}\left(Y_{R}>h+1\right)+\frac{1}{2} \mathbf{P}\left(Y_{R}=h+1\right)$

$$
=\mathbf{P}\left(X_{R}>h+1\right)+\mathbf{P}\left(X_{R}=h+1\right) z+\frac{1}{2}\left[\mathbf{P}\left(X_{R}=h+1\right)(1-z)+\mathbf{P}\left(X_{R}=h\right) z\right] .
$$

Moreover, note that $\mathbf{P}\left(X_{R}=h\right) \cdot z=\left(\begin{array}{c}2 h+1 \\ h\end{array}\right)(1-z)^{h+1} z^{h+1}=\mathbf{P}\left(X_{R}=h+1\right) \cdot(1-z)$. Therefore we conclude the proof by applying the latter equivalence into the previous formulation of $\mathbf{P}\left(\mathcal{E}_{2 h+2}\right)$, getting that $\mathbf{P}\left(\mathcal{E}_{2 h+1}\right)=\mathbf{P}\left(\mathcal{E}_{2 h+2}\right)$. 


\section{Proofs of Section 6}

\subsection{Proof of Proposition 6.1}

Proof. Let us compute, for each node $u \in V$, an upper bound to the expected fraction of neighbors in state $\mathcal{R}$ in round $t+1$ conditioned on the current configuration of states, namely

$$
\mathbf{E}\left[\phi_{u}^{(t+1)} \mid \mathbf{x}^{(t)}=\overline{\mathbf{x}}\right]=\frac{1}{\delta_{v}} \sum_{v \in N_{u}} \mathbf{P}\left(\left|\bar{R}_{v}^{(t)}\right|=1 \mid \mathbf{x}^{(t)}=\overline{\mathbf{x}}\right)=\frac{1}{\delta_{v}} \sum_{v \in N_{u}}(1-p) \phi_{v}^{(t)} \leq(1-p) \phi_{\max }^{(t)}
$$

Note that, in the previous equation, the conditioning exactly determines the fraction of neighbors in state $\mathcal{R}$ of every node in round $t$; thus we can compute the probability of every node to be in state $\mathcal{R}$ in the next round. Indeed, taking into account the effect of the bias, $\mathbf{P}\left(\left|\bar{R}_{v}^{(t)}\right|=1 \mid \mathbf{x}^{(t)}=\overline{\mathbf{x}}\right)=(1-p) \phi_{v}^{(t)}$, where $\phi_{v}^{(t)}$ is deterministic due to the conditioning.

As done in the proof of Proposition 5.5 for the fast convergence regime, with an application of a multiplicative form of the Chernoff Bound [DP09, Exercise 1.1] we get that

$$
\mathbf{P}\left(\phi_{u}^{(t+1)}>\left(1-p^{2}\right) \phi_{\max }^{(t)} \mid \mathbf{x}^{(t)}=\overline{\mathbf{x}}\right)=n^{-\omega(1)}
$$

and thus a union bound over all the agents allows us to claim that

$$
\mathbf{P}\left(\phi_{\max }^{(t+1)} \leq\left(1-p^{2}\right) \phi_{\max }^{(t)} \mid \mathbf{x}^{(t)}=\overline{\mathbf{x}}\right)=1-n^{-\omega(1)} .
$$

Therefore, since any initial configuration $\mathbf{x}^{(0)}$ is such that $\phi_{\max }^{(0)} \leq 1$, in round $t$ we have that $\phi_{u}^{(t)} \leq\left(1-p^{2}\right)^{t}$ which implies the thesis.

\subsection{Proofs of Proposition 6.2 and Proposition 6.3}

Proof of Proposition 6.2. Assume $p=\frac{1}{2}+c$. Called $Y$ the number of vertices which are in state $\mathcal{B}$ at round 1 , we notice that

$$
Y \sim \sum_{v \in V} X_{v}
$$

where $\left\{X_{v}\right\}_{v \in V}$ are independent random variables with $X_{v} \sim \operatorname{Bin}\left(1, r_{v}\right)$ and

$$
r_{v}:=\mathbf{P}\left(\operatorname{Bin}\left(\delta_{v}, p\right)>\frac{\delta_{v}}{2}=\delta_{v}(p-c)\right) \geq 1-e^{-\Theta\left(\delta_{v}\right)}
$$

hence, uniformly in $v \in V$,

$$
r_{v}=1-e^{-\omega(\log (n))}
$$

Therefore, by a union bound

$$
\mathbf{P}(Y=n) \geq 1-n e^{-\omega(\log (n))}=1-o(1) .
$$

Similarly, assume that $p=\frac{1}{2}-c$. Called $Z$ the number of vertices which are in state $\mathcal{R}$ at round 1 , we notice that

$$
Z \sim \sum_{v \in V} W_{v}
$$

where $W_{v} \sim \operatorname{Bin}\left(1, \ell_{v}\right)$

$$
\ell_{v}=\mathbf{P}\left(\operatorname{Bin}\left(\delta_{v}, p\right) \leq \frac{\delta_{v}}{2}=\delta_{v}(p+c)\right) \geq 1-e^{-\Theta\left(\delta_{v}\right)},
$$


hence, uniformly in $v \in V$,

$$
\ell_{v}=1-e^{-\omega(\log (n))} .
$$

Therefore, by a union bound

$$
z:=\mathbf{P}\left(\left|R^{(1)}\right|=n\right) \geq 1-n e^{-\omega(\log (n))} .
$$

Call $\tau$ the first $t \geq 0$ such that $B^{(t)} \neq \emptyset$, in other words,

$$
\mathbf{P}\left(\forall t \leq n^{K}, R^{(t)}=V\right)=\mathbf{P}\left(\tau>n^{K}\right) .
$$

Notice that $\tau$ has a geometric distribution of parameter $\mathbf{P}\left(\left|R^{(1)}\right| \neq n\right)$. Hence

$$
\mathbf{P}(\tau>t)=\mathbf{P}\left(\left|R^{(1)}\right|=n\right)^{t}=z^{t} .
$$

For every choice of $K>0$ independent of $n$, we can bound

$$
z^{t} \geq\left(1-n^{-2 K}\right)^{t} \sim e^{-t / n^{2 K}},
$$

from which the claim follows.

Proof of Proposition 6.3. We first prove Eq. 10. Fix $c>0$ and $p=\frac{1}{2}+c$. Assume that at round 0 all the vertices are in the state $\mathcal{R}$. Then

$$
\mathbf{P}_{k}\left(\phi_{v}^{(1)}>\gamma\right)=\mathbf{P}\left(\sum_{w \in V} X_{w}>\gamma \delta_{v}\right),
$$

where $\left\{X_{w}\right\}_{w \in V}$ are i.i.d. random variables with $X_{w} \sim \operatorname{Bin}\left(1, r_{k}\right)$ where

$$
\begin{aligned}
r_{k} & :=\mathbf{P}\left(\operatorname{Bin}(k, 1-p) \geq \frac{k+1}{2}\right) \\
& =\mathbf{P}\left(\operatorname{Bin}(k, 1-p) \geq k\left(\frac{1}{2}-c\right)\left(\frac{1}{1-2 c}+\frac{1}{k(1-2 c)}\right)\right) \leq e^{-M(c) k},
\end{aligned}
$$

for some constant $M(c)>0$. Hence

$$
\mathbf{P}\left(\sum_{w \in V} X_{w}>\gamma \delta_{v}\right)=\mathbf{P}\left(\operatorname{Bin}\left(\delta_{v}, r_{k}\right)>\gamma \delta_{v}\right)=\mathbf{P}\left(\operatorname{Bin}\left(\delta_{v}, r_{k}\right)>r_{k} \delta_{v}(1+\lambda)\right),
$$

where $\lambda=\frac{\gamma}{r_{k}}-1$. Since $r_{k}<e^{-M(c) k}$, there exists $\tilde{K}=\tilde{K}(c, \gamma)$ such that for $k>\tilde{K}$ we have $\gamma>r_{k}$, hence $\lambda>0$. Hence applying the Chernoff bound we get

$$
\mathbf{P}\left(\operatorname{Bin}\left(\delta_{v}, r_{k}\right)>r_{k} \delta_{v}(1+\lambda)\right) \leq e^{-\Theta\left(\delta_{v}\right)},
$$

uniformly in $k>\tilde{K}$. We can hence apply the union bounds over $v \in V$ and $t \in \operatorname{poly}(n)$.

Let us now prove Eq. [11. Fix $c>0, p=\frac{1}{2}-c$ and $\gamma>0$. By Lemma 8.3 and Proposition 5.2 with $p=\frac{1}{2}-c$, there exists $\bar{K}(c)>0$ such that for any fixed $K>0$ and for all $k>\bar{K}(c)$ it holds

$$
\mathbf{P}_{k}\left(\forall t<n^{K}, \quad \frac{\operatorname{vol}\left(R^{(t)}\right)}{\operatorname{vol}(V)} \geq \varphi_{k, \frac{1}{2}-c}^{+}-\frac{\gamma}{2}\right)=1-o(1)
$$


Note that, since $\varphi_{k, \frac{1}{2}-c}^{+} \rightarrow 1$ when $k \rightarrow+\infty$, we have that for any $\gamma>0$ there exists $\hat{K}(c, \gamma)$ such that for all $k>\hat{K}(c, \gamma)$ it holds

$$
1-\varphi_{k, \frac{1}{2}-c}^{+}<\frac{\gamma}{2}
$$

So by Eqs. (47) and (48) we get that, fixed $p=\frac{1}{2}-c$ and any $\gamma \in(0,1)$, for all $k:=$ $\max \{\bar{K}(c), \hat{K}(c, \gamma)\}$ we have that Eq. 11 holds.

The thesis follows by choosing $H(c, \gamma):=\max \{\bar{K}(c), \hat{K}(c, \gamma), \tilde{K}(c, \gamma)\}$.

\section{References}

[ABB19] Gideon Amir, Rangel Baldasso, and Nissan Beilin. Majority dynamics and the median process: connections, convergence and some new conjectures. arXiv preprint arXiv:1911.08613, 2019.

$\left[\mathrm{ABC}^{+} 20\right]$ Aris Anagnostopoulos, Luca Becchetti, Emilio Cruciani, Francesco Pasquale, and Sara Rizzo. Biased opinion dynamics: When the devil is in the details. In Christian Bessiere, editor, Proceedings of the Twenty-Ninth International Joint Conference on Artificial Intelligence, IJCAI 2020, pages 53-59. ijcai.org, 2020.

[ABF15] Mohammed Amin Abdullah, Michel Bode, and Nikolaos Fountoulakis. Local majority dynamics on preferential attachment graphs. In David F. Gleich, Júlia Komjáthy, and Nelly Litvak, editors, Algorithms and Models for the Web Graph - 12th International Workshop, WAW 2015, Eindhoven, The Netherlands, December 10-11, 2015, Proceedings, volume 9479 of Lecture Notes in Computer Science, pages 95-106. Springer, 2015.

[AD15] Mohammed Amin Abdullah and Moez Draief. Global majority consensus by local majority polling on graphs of a given degree sequence. Discret. Appl. Math., 180:1$10,2015$.

[AFG18] Vincenzo Auletta, Diodato Ferraioli, and Gianluigi Greco. Reasoning about consensus when opinions diffuse through majority dynamics. In Jérôme Lang, editor, Proceedings of the Twenty-Seventh International Joint Conference on Artificial Intelligence, IJCAI 2018, July 13-19, 2018, Stockholm, Sweden, pages 49-55. ijcai.org, 2018.

[BCE $\left.{ }^{+} 17\right]$ Petra Berenbrink, Andrea E. F. Clementi, Robert Elsässer, Peter Kling, Frederik Mallmann-Trenn, and Emanuele Natale. Ignore or comply?: On breaking symmetry in consensus. In Elad Michael Schiller and Alexander A. Schwarzmann, editors, Proceedings of the ACM Symposium on Principles of Distributed Computing, PODC 2017, Washington, DC, USA, July 25-27, 2017, pages 335-344. ACM, 2017.

$\left[\mathrm{BCN}^{+} 16\right]$ Luca Becchetti, Andrea E. F. Clementi, Emanuele Natale, Francesco Pasquale, and Luca Trevisan. Stabilizing consensus with many opinions. In Robert Krauthgamer, editor, Proceedings of the Twenty-Seventh Annual ACM-SIAM Symposium on Discrete Algorithms, SODA 2016, Arlington, VA, USA, January 10-12, 2016, pages 620-635. SIAM, 2016.

$\left[\mathrm{BCN}^{+} 17\right]$ Luca Becchetti, Andrea E. F. Clementi, Emanuele Natale, Francesco Pasquale, Riccardo Silvestri, and Luca Trevisan. Simple dynamics for plurality consensus. Distributed Computing, 30(4):293-306, 2017. 
[BCN20] Luca Becchetti, Andrea Clementi, and Emanuele Natale. Consensus dynamics: An overview. SIGACT News, 51(1):58104, March 2020.

$\left[\mathrm{BCO}^{+} 16\right]$ Itai Benjamini, Siu-On Chan, Ryan ODonnell, Omer Tamuz, and Li-Yang Tan. Convergence, unanimity and disagreement in majority dynamics on unimodular graphs and random graphs. Stochastic Processes and their Applications, 126(9):2719-2733, 2016.

[BCPR19] Luca Becchetti, Emilio Cruciani, Francesco Pasquale, and Sara Rizzo. Step-by-step community detection in volume-regular graphs. In 30th International Symposium on Algorithms and Computation, ISAAC 2019, December 8-11, 2019, Shanghai University of Finance and Economics, Shanghai, China, volume 149 of LIPIcs, pages 20:1-20:23. Schloss Dagstuhl - Leibniz-Zentrum für Informatik, 2019.

[BGKM16] Petra Berenbrink, George Giakkoupis, Anne-Marie Kermarrec, and Frederik Mallmann-Trenn. Bounds on the voter model in dynamic networks. In Ioannis Chatzigiannakis, Michael Mitzenmacher, Yuval Rabani, and Davide Sangiorgi, editors, 43rd International Colloquium on Automata, Languages, and Programming, ICALP 2016, July 11-15, 2016, Rome, Italy, volume 55 of LIPIcs, pages 146:1146:15. Schloss Dagstuhl - Leibniz-Zentrum für Informatik, 2016.

[BIMW20] Maryam Bahrani, Nicole Immorlica, Divyarthi Mohan, and S. Matthew Weinberg. Asynchronous majority dynamics in preferential attachment trees. In 4 7th International Colloquium on Automata, Languages, and Programming, ICALP 2020 (To appear), 2020.

[CEOR13] Colin Cooper, Robert Elsässer, Hirotaka Ono, and Tomasz Radzik. Coalescing random walks and voting on connected graphs. SIAM J. Discrete Math., 27(4):1748$1758,2013$.

[CER14] Colin Cooper, Robert Elsässer, and Tomasz Radzik. The power of two choices in distributed voting. In Javier Esparza, Pierre Fraigniaud, Thore Husfeldt, and Elias Koutsoupias, editors, Automata, Languages, and Programming - 41st International Colloquium, ICALP 2014, Copenhagen, Denmark, July 8-11, 2014, Proceedings, Part II, volume 8573 of Lecture Notes in Computer Science, pages 435-446. Springer, 2014.

$\left[\mathrm{CER}^{+} 15\right]$ Colin Cooper, Robert Elsässer, Tomasz Radzik, Nicolas Rivera, and Takeharu Shiraga. Fast consensus for voting on general expander graphs. In Yoram Moses, editor, Distributed Computing - 29th International Symposium, DISC 2015, Tokyo, Japan, October 7-9, 2015, Proceedings, volume 9363 of Lecture Notes in Computer Science, pages 248-262. Springer, 2015.

[CGG $\left.{ }^{+} 18\right]$ Andrea E. F. Clementi, Mohsen Ghaffari, Luciano Gualà, Emanuele Natale, Francesco Pasquale, and Giacomo Scornavacca. A tight analysis of the parallel undecided-state dynamics with two colors. In Igor Potapov, Paul G. Spirakis, and James Worrell, editors, 43rd International Symposium on Mathematical Foundations of Computer Science, MFCS 2018, August 27-31, 2018, Liverpool, UK, volume 117 of LIPIcs, pages 28:1-28:15. Schloss Dagstuhl - Leibniz-Zentrum fuer Informatik, 2018.

[CNNS18] Emilio Cruciani, Emanuele Natale, André Nusser, and Giacomo Scornavacca. Phase transition of the 2-choices dynamics on core-periphery networks. In Elisabeth André, 
Sven Koenig, Mehdi Dastani, and Gita Sukthankar, editors, Proceedings of the 17th International Conference on Autonomous Agents and MultiAgent Systems, AAMAS 2018, Stockholm, Sweden, July 10-15, 2018, pages 777-785. International Foundation for Autonomous Agents and Multiagent Systems Richland, SC, USA / ACM, 2018 .

[CNS19] Emilio Cruciani, Emanuele Natale, and Giacomo Scornavacca. Distributed community detection via metastability of the 2-choices dynamics. In The Thirty-Third AAAI Conference on Artificial Intelligence, AAAI 2019, Honolulu, Hawaii, USA, January 27 - February 1, 2019, pages 6046-6053. AAAI Press, 2019.

[CRRS17] Colin Cooper, Tomasz Radzik, Nicolas Rivera, and Takeharu Shiraga. Fast plurality consensus in regular expanders. In Andréa W. Richa, editor, 31st International Symposium on Distributed Computing, DISC 2017, October 16-20, 2017, Vienna, Austria, volume 91 of LIPIcs, pages 13:1-13:16. Schloss Dagstuhl - Leibniz-Zentrum fuer Informatik, 2017.

[DeG74] Morris H DeGroot. Reaching a consensus. Journal of the American Statistical Association, 69(345):118-121, 1974.

[DGM $\left.{ }^{+} 11\right]$ Benjamin Doerr, Leslie Ann Goldberg, Lorenz Minder, Thomas Sauerwald, and Christian Scheideler. Stabilizing consensus with the power of two choices. In Rajmohan Rajaraman and Friedhelm Meyer auf der Heide, editors, SPAA 2011: Proceedings of the 23rd Annual ACM Symposium on Parallelism in Algorithms and Architectures, San Jose, CA, USA, June 4-6, 2011 (Co-located with FCRC 2011), pages 149-158. ACM, 2011.

[DP09] Devdatt P. Dubhashi and Alessandro Panconesi. Concentration of Measure for the Analysis of Randomized Algorithms. Cambridge University Press, 2009.

[DW83] Peter Donnelly and Dominic Welsh. Finite particle systems and infection models. In Mathematical Proceedings of the Cambridge Philosophical Society, volume 94, pages 167-182, 1983.

[EFK $\left.{ }^{+} 17\right]$ Robert Elsässer, Tom Friedetzky, Dominik Kaaser, Frederik Mallmann-Trenn, and Horst Trinker. Brief announcement: Rapid asynchronous plurality consensus. In Elad Michael Schiller and Alexander A. Schwarzmann, editors, Proceedings of the ACM Symposium on Principles of Distributed Computing, PODC 2017, Washington, DC, USA, July 25-27, 2017, pages 363-365. ACM, 2017.

[FILW14] Michal Feldman, Nicole Immorlica, Brendan Lucier, and S. Matthew Weinberg. Reaching consensus via non-bayesian asynchronous learning in social networks. In Klaus Jansen, José D. P. Rolim, Nikhil R. Devanur, and Cristopher Moore, editors, Approximation, Randomization, and Combinatorial Optimization. Algorithms and Techniques, APPROX/RANDOM 2014, September 4-6, 2014, Barcelona, Spain, volume 28 of LIPIcs, pages 192-208. Schloss Dagstuhl - Leibniz-Zentrum für Informatik, 2014.

[FJ90] Noah E Friedkin and Eugene C Johnsen. Social influence and opinions. Journal of Mathematical Sociology, 15(3-4):193-206, 1990.

[FN19] Pierre Fraigniaud and Emanuele Natale. Noisy rumor spreading and plurality consensus. Distributed Computing, 32(4):257-276, 2019. 
[GL18] Mohsen Ghaffari and Johannes Lengler. Nearly-tight analysis for 2-choice and 3majority consensus dynamics. In Calvin Newport and Idit Keidar, editors, Proceedings of the 2018 ACM Symposium on Principles of Distributed Computing, PODC 2018, Egham, United Kingdom, July 23-27, 2018, pages 305-313. ACM, 2018.

[GZ18] Bernd Gärtner and Ahad N. Zehmakan. Majority model on random regular graphs. In Michael A. Bender, Martin Farach-Colton, and Miguel A. Mosteiro, editors, LATIN 2018: Theoretical Informatics - 13th Latin American Symposium, Buenos Aires, Argentina, April 16-19, 2018, Proceedings, volume 10807 of Lecture Notes in Computer Science, pages 572-583. Springer, 2018.

$\left[\mathrm{HL}^{+} 75\right]$ Richard A Holley, Thomas M Liggett, et al. Ergodic theorems for weakly interacting infinite systems and the voter model. The annals of probability, 3(4):643-663, 1975.

[HLL83] Paul W Holland, Kathryn Blackmond Laskey, and Samuel Leinhardt. Stochastic blockmodels: First steps. Social networks, 5(2):109-137, 1983.

[HP01] Yehuda Hassin and David Peleg. Distributed probabilistic polling and applications to proportionate agreement. Inf. Comput., 171(2):248-268, 2001.

[KMS19] Varun Kanade, Frederik Mallmann-Trenn, and Thomas Sauerwald. On coalescence time in graphs: When is coalescing as fast as meeting?: Extended abstract. In Timothy M. Chan, editor, Proceedings of the Thirtieth Annual ACM-SIAM Symposium on Discrete Algorithms, SODA 2019, San Diego, California, USA, January 6-9, 2019, pages 956-965. SIAM, 2019.

[KR03] Paul L Krapivsky and Sidney Redner. Dynamics of majority rule in two-state interacting spin systems. Physical Review Letters, 90(23):238701, 2003.

[KR19] Nan Kang and Nicolás Rivera. Best-of-three voting on dense graphs. In The 31st ACM Symposium on Parallelism in Algorithms and Architectures, SPAA 19, page 115121, New York, NY, USA, 2019. Association for Computing Machinery.

[KS60] John G. Kemeny and J. Laurie Snell. Finite Markov chains. D. van Nostrand Company, inc., Princeton, N.J., 1960.

[Lig12] Thomas Milton Liggett. Interacting particle systems, volume 276. Springer Science \& Business Media, 2012.

[MBCD19] Wenjun Mei, Francesco Bullo, Ge Chen, and Florian Dörfler. Occam's razor in opinion dynamics: The weighted-median influence process. CoRR, abs/1909.06474, 2019 .

[MMR16] A. Mukhopadhyay, R. R. Mazumdar, and R. Roy. Binary opinion dynamics with biased agents and agents with different degrees of stubbornness. In International Teletraffic Congress, volume 01, pages 261-269, 2016.

[MNT14] Elchanan Mossel, Joe Neeman, and Omer Tamuz. Majority dynamics and aggregation of information in social networks. Autonomous Agents and Multi-Agent Systems, 28(3):408-429, 2014.

[MT17] Elchanan Mossel and Omer Tamuz. Opinion exchange dynamics. Probab. Surveys, 14:155-204, 2017. 
[SLST17] Alina Sîrbu, Vittorio Loreto, Vito D. P. Servedio, and Francesca Tria. Opinion dynamics: Models, extensions and external effects. In Vittorio Loreto, Muki Haklay, Andreas Hotho, Vito D. P. Servedio, Gerd Stumme, Jan Theunis, and Francesca Tria, editors, Participatory Sensing, Opinions and Collective Awareness, Understanding Complex Systems, pages 363-401. Springer, 2017.

[SS19] Nobutaka Shimizu and Takeharu Shiraga. Phase transitions of best-of-two and best-of-three on stochastic block models. In Jukka Suomela, editor, 33rd International Symposium on Distributed Computing, DISC 2019, October 14-18, 2019, Budapest, Hungary, volume 146 of LIPIcs, pages 32:1-32:17. Schloss Dagstuhl Leibniz-Zentrum für Informatik, 2019.

[SS20] Nobutaka Shimizu and Takeharu Shiraga. Quasi-majority functional voting on expander graphs. In 47 th International Colloquium on Automata, Languages, and Programming, ICALP 2020 (To appear), 2020.

[Zeh20] Ahad N. Zehmakan. Opinion forming in erdős-rényi random graph and expanders. Discret. Appl. Math., 277:280-290, 2020. 